\title{
Existence of Asymptotically Almost Automorphic Mild Solutions of Semilinear Fractional Differential Equations
}

\author{
Junfei Cao, ${ }^{1}$ Zaitang Huang $\mathbb{D}^{2},{ }^{2}$ and Gaston M. N'Guérékata $\mathbb{D}^{3}$ \\ ${ }^{1}$ Department of Mathematics, Guangdong University of Education, Guangzhou 510303, China \\ ${ }^{2}$ School of Mathematical Sciences, Guangxi Teachers Education University, Nanning 530023, China \\ ${ }^{3}$ Department of Mathematics, Morgan State University, Baltimore, MD 21251, USA
}

Correspondence should be addressed to Gaston M. N’Guérékata; nguerekata@aol.com

Received 21 December 2017; Revised 18 April 2018; Accepted 10 May 2018; Published 1 August 2018

Academic Editor: Patricia J. Y. Wong

Copyright (C) 2018 Junfei Cao et al. This is an open access article distributed under the Creative Commons Attribution License, which permits unrestricted use, distribution, and reproduction in any medium, provided the original work is properly cited.

\begin{abstract}
This paper is concerned with the existence of asymptotically almost automorphic mild solutions to a class of abstract semilinear fractional differential equations $\mathrm{D}_{t}^{\alpha} x(t)=A x(t)+\mathrm{D}_{t}^{\alpha-1} F(t, x(t), B x(t)), t \in \mathbb{R}$, where $1<\alpha<2, A$ is a linear densely defined operator of sectorial type on a complex Banach space $X$ and $B$ is a bounded linear operator defined on $X, F$ is an appropriate function defined on phase space, and the fractional derivative is understood in the Riemann-Liouville sense. Combining the fixed point theorem due to Krasnoselskii and a decomposition technique, we prove the existence of asymptotically almost automorphic mild solutions to such problems. Our results generalize and improve some previous results since the (locally) Lipschitz continuity on the nonlinearity $F$ is not required. The results obtained are utilized to study the existence of asymptotically almost automorphic mild solutions to a fractional relaxation-oscillation equation.
\end{abstract}

\section{Introduction}

The almost periodic function introduced seminally by Bohr in 1925 plays an important role in describing the phenomena that are similar to the periodic oscillations which can be observed frequently in many fields, such as celestial mechanics, nonlinear vibration, electromagnetic theory, plasma physics, engineering, and ecosphere. The concept of almost automorphy, which is an important generalization of the classical almost periodicity, was first introduced in the literature [1-4] by Bochner in relation to some aspects of differential geometry. Since then, this pioneer work has attracted more and more attention and has been substantially extended in several different directions. Many authors have made important contributions to this theory (see, for instance, [5-17] and the references therein). Especially, in [5, 6], the authors gave an important overview about the theory of almost automorphic functions and their applications to differential equations.

As a natural extension of almost automorphy, the concept of asymptotic almost automorphy, which is the central issue to be discussed in this paper, was introduced in the literature [18] by N'Guérékata in the early eighties. Since then, this notion has found several developments and has been generalized into different directions. Until now, the asymptotically almost automorphic functions as well as the asymptotically almost automorphic solutions for differential systems have been investigated by many mathematicians; see [19] by Bugajewski and N'Guérékata, [20] by Diagana, Hernández, and dos Santos, and [21] by Ding, Xiao, and Liang for the asymptotically almost automorphic solutions to integrodifferential equations, see [22] by Zhao, Chang, and N'Guérékata for the asymptotically almost automorphic solutions to the nonlinear delay integral equations, and see [23] by Chang and Tang and [24] by Zhao, Chang, and Nieto for the asymptotically almost automorphic solutions to stochastic differential equations, and the existence of asymptotically almost automorphic solutions has become one of the most attractive topics in the qualitative theory of differential equations due to its significance and applications in physics, mathematical biology, control theory, and so on. We refer the reader to the monographs of N'Guérékata [25] for the recently theory and applications of asymptotically almost automorphic functions. 
With motivation coming from a wide range of engineering and physical applications, fractional differential equations have recently attracted great attention of mathematicians and scientists. This kind of equations is a generalization of ordinary differential equations to arbitrary noninteger orders. Fractional differential equations find numerous applications in the field of viscoelasticity, feedback amplifiers, electrical circuits, electro analytical chemistry, fractional multipoles, neuron modelling encompassing different branches of physics, chemistry, and biological sciences [26-32]. Many physical processes appear to exhibit fractional order behavior that may vary with time or space. In recent years, there has been a significant development in ordinary and partial differential equations involving fractional derivatives; we only enumerate here the monographs of Kilbas et al. [26, 27], Diethelm [28], Hilfer [29], Podlubny [30], Miller [31], and Zhou [32] and the papers of Agarwal et al. [33, 34], Benchohra et al. [35, 36], El-Borai [37], Lakshmikantham et al. [38-41], Mophou et al. [42-45], N'Guérékata [46], and Zhou et al. [4750] and the reference therein.

The study of almost periodic and almost automorphic type solutions to fractional differential equations was initiated by Araya and Lizama [11]. In their work, the authors investigated the existence and uniqueness of an almost automorphic mild solution of the semilinear fractional differential equation

$$
\mathrm{D}_{t}^{\alpha} x(t)=A x(t)+F(t, x(t)), \quad t \in \mathbb{R}, 1<\alpha<2,
$$

when $A$ is a generator of an $\alpha$-resolvent family and $\mathrm{D}_{t}^{\alpha}$ is the Riemann-Liouville fractional derivative. In [51], Cuevas and Lizama considered the fractional differential equation:

$$
\begin{aligned}
& \mathrm{D}_{t}^{\alpha} x(t)=A x(t)+\mathrm{D}_{t}^{\alpha-1} F(t, x(t)), \\
& \\
& t \in \mathbb{R}, 1<\alpha<2,
\end{aligned}
$$

where $A$ is a linear operator of sectorial negative type on a complex Banach space $X$ and the fractional derivative is understood in the Riemann-Liouville sense. Under suitable conditions on $F(t, x)$, the authors proved the existence and uniqueness of an almost automorphic mild solution to (2). Cuevas et al. [52, 53] studied, respectively, the pseudo almost periodic and pseudo almost periodic class infinity mild solutions to (2) assuming that $F: \mathbb{R} \times X \longrightarrow X$ and $(t, x) \longrightarrow F(t, x)$ is a pseudo almost periodic and pseudo almost periodic of class infinity function satisfying suitable conditions in $x \in X$. Agarwal et al. [54] studied the existence and uniqueness of a weighted pseudo almost periodic mild solution to equation (2). Ding et al. [55] investigated the existence and uniqueness of almost automorphic solution to (2) assuming that $F: \mathbb{R} \times X \longrightarrow X$ and $(t, x) \longrightarrow F(t, x)$ is Stepanov-like almost automorphic in $t \in \mathbb{R}$ satisfying some kind of Lipschitz conditions. Cuevas et al. [56] studied the existence of almost periodic (resp., pseudo almost periodic) mild solutions to equation (2) assuming that $F: \mathbb{R} \times X \longrightarrow X$ and $(t, x) \longrightarrow F(t, x)$ is Stepanov almost (resp., Stepanovlike pseudo almost) periodic in $t \in \mathbb{R}$ uniformly for $x \in X$. Chang et al. [57] studied the existence and uniqueness of weighted pseudo almost automorphic solution to equation
(2) with Stepanov-like weighted pseudo almost automorphic coefficient. He et al. [58] studied also the existence and uniqueness of weighted Stepanov-like pseudo almost automorphic mild solution to (2). Cao et al. [59] studied the existence and uniqueness of antiperiodic mild solution to (2). In [60], Cuevas et al. showed sufficient conditions to ensure the existence and uniqueness of mild solution for (2) in the following classes of vector-valued function spaces: periodic functions, asymptotically periodic functions, pseudo periodic functions, almost periodic functions, asymptotically almost periodic functions, pseudo almost periodic functions, almost automorphic functions, asymptotically almost automorphic functions, pseudo almost automorphic functions, compact almost automorphic functions, asymptotically compact almost automorphic functions, pseudo compact almost automorphic functions, $S$-asymptotically $\omega$-periodic functions, decay functions, and mean decay functions.

Recently, Xia et al. [61] established some sufficient criteria for the existence and uniqueness of $(\mu, \nu)$-pseudo almost automorphic solution to the semilinear fractional differential equation

$$
\mathrm{D}_{t}^{\alpha} x(t)=A x(t)+\mathrm{D}_{t}^{\alpha-1} F(t, B x(t)), \quad t \in \mathbb{R},
$$

where $1<\alpha<2, A$ is a sectorial operator of type $\omega<0$ on a complex Banach space $X$ and $B$ is a bounded linear operator. The fractional derivative is understood in the RiemannLiouville sense. Their discussion is divided into two cases, i.e., $F: \mathbb{R} \times X \longrightarrow X,(t, x) \longrightarrow F(t, x)$ is $(\mu, v)$-pseudo almost automorphic and $F: \mathbb{R} \times X \longrightarrow X$, and $(t, x) \longrightarrow F(t, x)$ is Stepanov-like $(\mu, v)$-pseudo almost automorphic. Kavitha et al. [62] studied weighted pseudo almost automorphic solutions of the fractional integrodifferential equation

$$
\mathrm{D}_{t}^{\alpha} x(t)=A x(t)+\mathrm{D}_{t}^{\alpha-1} F(t, x(t), K x(t)), \quad t \in \mathbb{R},
$$

where $1<\alpha<2$ and

$$
K x(t)=\int_{-\infty}^{t} k(t-s) h(s, x(s)) \mathrm{d} s,
$$

$A$ is a linear densely defined sectorial operator on a complex Banach space $X, F: \mathbb{R} \times X \times X \longrightarrow X$, and $(t, x, y) \longrightarrow$ $F(t, x, y)$ is a weighted pseudo almost automorphic function in $t \in \mathbb{R}$ for each $x, y \in X$ satisfying suitable conditions. The fractional derivative is understood in the RiemannLiouville sense. Mophou [63] investigated the existence and uniqueness of weighted pseudo almost automorphic mild solution to the fractional differential equation:

$$
\begin{aligned}
\mathrm{D}_{t}^{\alpha} x(t)=A x(t)+\mathrm{D}_{t}^{\alpha-1} F(t, x(t), & B x(t)), \\
& \\
t & \in \mathbb{R}, 1<\alpha<2,
\end{aligned}
$$

where $A: D(A) \subset X \longrightarrow X$ is a linear densely operator of sectorial type on a complex Banach space $X, B$ : $X \longrightarrow X$ is a bounded linear operator and $F: \mathbb{R} \times X \times$ $X \longrightarrow X$, and $(t, x, y) \longrightarrow F(t, x, y)$ is a weighted pseudo almost automorphic function in $t \in \mathbb{R}$ for each $x, y \in X$ satisfying suitable conditions. The fractional derivative $\mathrm{D}_{t}^{\alpha}$ is to be understood in Riemann-Liouville sense. Chang et al. 
[64] investigated some existence results of $\mu$-pseudo almost automorphic mild solutions to (6) assuming that $F: \mathbb{R} \times X \times$ $X \longrightarrow X$ and $(t, x, y) \longrightarrow F(t, x, y)$ is a $\mu$-pseudo almost automorphic function in $t \in \mathbb{R}$ for each $x, y \in X$ satisfying suitable conditions. For more on the almost periodicity and almost automorphy for fractional differential equations and related issues, we refer the reader to [65-67] and others.

Equation (6) is motivated by physical problems. Indeed, due to their applications in fields of science where characteristics of anomalous diffusion are presented, type (6) equations are attracting increasing interest (cf. [68-70] and references therein). For example, anomalous diffusion in fractals [69] or in macroeconomics [71] has been recently well studied in the setting of fractional Cauchy problems like (6). For this reason, (6) has gotten a considerable attention in recent years (cf. [51$64,68-71$ ] and the references therein).

To the best of our knowledge, much less is known about the existence of asymptotically almost automorphic mild solutions to (6) when the nonlinearity $F(t, x, y)$ as a whole loses the Lipschitz continuity with respect to $x$ and $y$. Motivated by the abovementioned works, the purpose of this paper is to establish some new existence results of asymptotically almost automorphic mild solutions to (6). In our results, the nonlinearity $F: \mathbb{R} \times X \times X \longrightarrow$ $X,(t, x, y) \longrightarrow F(t, x, y)$ does not have to satisfy a (locally) Lipschitz condition (see Remark 22). However, in many papers (for instance, $[11,51-64]$ ) on almost periodic type and almost automorphic type solutions to fractional differential equations, to be able to apply the well-known Banach contraction principle, a (locally) Lipschitz condition for the nonlinearity of corresponding fractional differential equations is needed. As can be seen, our results generalize those as well as related research and have more broad applications. In particular, as application and to illustrate our main results, we will examine some sufficient conditions for the existence of asymptotically almost automorphic mild solutions to the fractional relaxation-oscillation equation given by

$$
\begin{aligned}
& \partial_{t}^{\alpha} u(t, x)=\partial_{x}^{2} u(t, x)-p u(t, x)+\partial_{t}^{\alpha-1}[\mu a(t) \\
& \cdot \sin \left(\frac{1}{2+\cos t+\cos \sqrt{2} t}\right)[\sin u(t, x)+u(t, x)] \\
& \left.\quad+v e^{-|t|}[u(t, x)+\sin u(t, x)]\right], \quad t \in \mathbb{R}, x \in[0, \pi]
\end{aligned}
$$

with boundary conditions $u(t, 0)=u(t, \pi)=0, t \in \mathbb{R}$, where $a(t) \in B C\left(\mathbb{R}, \mathbb{R}^{+}\right)$is a function and $p, \mu$, and $\nu$ are positive constants.

The rest of this paper is organized as follows. In Section 2, some concepts, the related notations, and some useful lemmas are introduced and established. In Section 3, we prove the existence of asymptotically almost automorphic mild solutions to such problems. The results obtained are utilized to study the existence of asymptotically almost automorphic mild solutions to a fractional relaxation-oscillation equation given in Section 4.

\section{Preliminaries}

This section is concerned with some notations, definitions, lemmas, and preliminary facts which are used in what follows.

From now on, let $(X,\|\cdot\|)$ and $\left(Y,\|\cdot\|_{Y}\right)$ be two Banach spaces and $B C(\mathbb{R}, X)$ (resp., $B C(\mathbb{R} \times Y \times Y, X))$ is the space of all $X$-valued bounded continuous functions (resp., jointly bounded continuous functions $F: \mathbb{R} \times Y \times Y \rightarrow X)$. Furthermore, $C_{0}(\mathbb{R}, X)$ (resp., $C_{0}(\mathbb{R} \times Y \times Y, X)$ ) is the closed subspace of $B C(\mathbb{R}, X)$ (resp., $B C(\mathbb{R} \times Y \times Y, X)$ ) consisting of functions vanishing at infinity (vanishing at infinity uniformly in any compact subset of $Y \times Y$, in other words,

$$
\lim _{|t| \longrightarrow+\infty}\|g(t, x, y)\|=0 \quad \text { uniformly for }(x, y) \in \mathbb{K},
$$

where $\mathbb{K}$ is an any compact subset of $Y \times Y)$. Let also $\mathbb{L}(X)$ be the Banach space of all bounded linear operators from $X$ into itself endowed with the norm:

$$
\|T\|_{\mathbb{L}(X)}=\sup \{\|T x\|: x \in X,\|x\|=1\} .
$$

For a bounded linear operator $A \in \mathbb{L}(X)$, let $\rho(A)$ and $D(A)$ stand for the resolvent and domain of $A$, respectively.

First, let us recall some basic definitions and results on almost automorphic and asymptotically almost automorphic functions.

Definition 1 ((Bochner) [1] (N’Guérékata) [6]). A continuous function $F: \mathbb{R} \longrightarrow X$ is said to be almost automorphic if for every sequence of real numbers $\left\{s_{n}^{\prime}\right\}$, there exists a subsequence $\left\{s_{n}\right\}$ such that

$$
\Theta(t)=\lim _{n \longrightarrow \infty} F\left(t+s_{n}\right)
$$

is well defined for each $t \in \mathbb{R}$ and

$$
\lim _{n \longrightarrow \infty} \Theta\left(t-s_{n}\right)=F(t) \quad \text { for each } t \in \mathbb{R} .
$$

Denote by $A A(\mathbb{R}, X)$ the set of all such functions.

Remark 2 (see [6]). By the point-wise convergence, the function $\Theta(t)$ in Definition 1 is measurable but not necessarily continuous. Moreover, if $\Theta(t)$ is continuous, then $F(t)$ is uniformly continuous (cf., e.g., [17], Theorem 2.6), and if the convergence in Definition 1 is uniform on $\mathbb{R}$, one gets almost periodicity (in the sense of Bochner and von Neumann). Almost automorphy is thus a more general concept than almost periodicity. There exists an almost automorphic function which is not almost periodic. The function $F: \mathbb{R} \longrightarrow$ $\mathbb{R}$ given by

$$
F(t)=\sin \left(\frac{1}{2+\cos t+\cos \sqrt{2} t}\right)
$$

is an example of such functions [72].

Lemma 3 (see [5]). $A A(\mathbb{R}, X)$ is a Banach space with the norm $\|F\|_{\infty}=\sup _{t \in \mathbb{R}}\|F(t)\|$. 
Definition 4 (see [6]). A continuous function $F: \mathbb{R} \times Y \times Y \longrightarrow$ $X$ is said to be almost automorphic in $t \in \mathbb{R}$ uniformly for all $(x, y) \in K$, where $K$ is any bounded subset of $Y \times Y$, if for every sequence of real numbers $\left\{s_{n}^{\prime}\right\}$, there exists a subsequence $\left\{s_{n}\right\}$ such that

$$
\lim _{n \longrightarrow \infty} F\left(t+s_{n}, x, y\right)=\Theta(t, x, y) \text { exists }
$$$$
\text { for each } t \in \mathbb{R} \text { and each }(x, y) \in K
$$

and

$$
\lim _{n \longrightarrow \infty} \Theta\left(t-s_{n}, x, y\right)=F(t, x, y) \text { exists }
$$

for each $t \in \mathbb{R}$ and each $(x, y) \in K$.

The collection of those functions is denoted by $A A(\mathbb{R} \times Y \times$ $Y, X)$.

Remark 5. The function $F: \mathbb{R} \times X \times X \longrightarrow X$ given by

$$
F(t, x, y)=\sin \left(\frac{1}{2+\cos t+\cos \sqrt{2} t}\right)[\sin (x)+y]
$$

is almost automorphic in $t \in \mathbb{R}$ uniformly for all $(x, y) \in K$, where $K$ is any bounded subset of $X \times X, X=L^{2}[0, \pi]$.

Similar to Lemma 2.2 of [73] and Proposition 3.2 of [63], we have the following result on almost automorphic functions.

Lemma 6. Let $F: \mathbb{R} \times X \times X \longrightarrow X$ be almost automorphic in $t \in \mathbb{R}$ uniformly for all $(x, y) \in K$, where $K$ is any bounded subset of $X \times X$, and assume that $F(t, x, y)$ is uniformly continuous on $K$ uniformly for $t \in \mathbb{R}$, that is, for any $\varepsilon>0$, there exists $\delta>0$ such that $x_{1}, x_{2}, y_{1}, y_{2} \in K$ and $\left\|x_{1}-y_{1}\right\|+$ $\left\|x_{2}-y_{2}\right\|<\delta$ imply that

$$
\left\|F\left(t, x_{1}, x_{2}\right)-F\left(t, y_{1}, y_{2}\right)\right\|<\varepsilon \quad \forall t \in \mathbb{R} .
$$

Let $x, y: \mathbb{R} \longrightarrow X$ be almost automorphic. Then the function $\Upsilon: \mathbb{R} \longrightarrow X$ defined by $\Upsilon(t)=F(t, x(t), y(t))$ is almost automorphic.

Proof. Suppose that $\left\{s_{n}\right\}$ is a sequence of real numbers. Then by the definition of almost automorphic functions, we can extract a subsequence $\left\{\tau_{n}\right\}$ of $\left\{s_{n}\right\}$ such that

$\left(P_{1}\right) \lim _{n \longrightarrow \infty} x\left(t+\tau_{n}\right)=\tilde{x}(t) \quad$ for each $t \in \mathbb{R}$,

$\left(P_{2}\right) \lim _{n \longrightarrow \infty} \tilde{x}\left(t-\tau_{n}\right)=x(t) \quad$ for each $t \in \mathbb{R}$,

$\left(P_{3}\right) \lim _{n \rightarrow \infty} y\left(t+\tau_{n}\right)=\tilde{y}(t) \quad$ for each $t \in \mathbb{R}$,

$\left(P_{4}\right) \lim _{n \longrightarrow \infty} \tilde{y}\left(t-\tau_{n}\right)=y(t) \quad$ for each $t \in \mathbb{R}$,

$\left(P_{5}\right) \lim _{n \longrightarrow \infty} F\left(t+\tau_{n}, x, y\right)=\widetilde{F}(t, x, y)$

for each $t \in \mathbb{R}, x, y \in X$,

$\left(P_{6}\right) \lim _{n \longrightarrow \infty} \widetilde{F}\left(t-\tau_{n}, x, y\right)=F(t, x, y)$

for each $t \in \mathbb{R}, x, y \in X$.
Write

$$
\tilde{\Upsilon}(t):=\widetilde{F}(t, \tilde{x}(t), \tilde{y}(t)), \quad t \in \mathbb{R} .
$$

Then

$$
\begin{aligned}
& \left\|\Upsilon\left(t+\tau_{n}\right)-\tilde{Y}(t)\right\| \\
& \quad=\| F\left(t+\tau_{n}, x\left(t+\tau_{n}\right), y\left(t+\tau_{n}\right)\right) \\
& \quad-\widetilde{F}(t, \tilde{x}(t), \tilde{y}(t)) \| \\
& \quad \leq \| F\left(t+\tau_{n}, x\left(t+\tau_{n}\right), y\left(t+\tau_{n}\right)\right) \\
& \quad-F\left(t+\tau_{n}, \tilde{x}(t), \tilde{y}(t)\right)\|+\| F\left(t+\tau_{n}, \tilde{x}(t), \tilde{y}(t)\right) \\
& \quad-\widetilde{F}(t, \tilde{x}(t), \tilde{y}(t)) \| .
\end{aligned}
$$

Since $x(t)$ and $y(t)$ are almost automorphic, then $x(t), y(t)$ and $\tilde{x}(t)$, and $\widetilde{y}(t)$ are bounded. Therefore we can choose a bounded subset $K \subset X \times X$, such that

$$
\begin{aligned}
& (x(t), y(t)) \in K, \\
& (\tilde{x}(t), \tilde{y}(t)) \in K
\end{aligned}
$$

$\forall t \in \mathbb{R}$

By $\left(P_{1}\right),\left(P_{3}\right)$, and the uniform continuity of $F(t, x, y)$ in $(x(t), y(t)) \in K$, we have

$$
\begin{gathered}
\lim _{n \rightarrow \infty} \| F\left(t+\tau_{n}, x\left(t+\tau_{n}\right), y\left(t+\tau_{n}\right)\right) \\
-F\left(t+\tau_{n}, \tilde{x}(t), \tilde{y}(t)\right) \|=0 .
\end{gathered}
$$

Moreover, by $\left(P_{5}\right)$,

$$
\lim _{n \longrightarrow \infty}\left\|F\left(t+\tau_{n}, \tilde{x}(t), \tilde{y}(t)\right)-\widetilde{F}(t, \tilde{x}(t), \tilde{y}(t))\right\|=0,
$$

so remembering the above triangle inequality, we deduce that

$$
\lim _{n \longrightarrow \infty}\left\|\Upsilon\left(t+\tau_{n}\right)-\tilde{\Upsilon}(t)\right\|=0 \quad \text { for each } t \in \mathbb{R} .
$$

Using the same argument we can prove that

$$
\lim _{n \longrightarrow \infty}\left\|\widetilde{\Upsilon}\left(t-\tau_{n}\right)-\Upsilon(t)\right\|=0 \quad \text { for each } t \in \mathbb{R} .
$$

This proves that $\Upsilon(t)$ is almost automorphic by the definition.

Remark 7. If $F(t, x, y)$ satisfies a Lipschitz condition with respect to $x$ and $y$ uniformly in $t \in \mathbb{R}$, i.e., for each pair $x_{1}, x_{2}, y_{1}, y_{2} \in X$,

$$
\begin{aligned}
& \left\|F\left(t, x_{1}, x_{2}\right)-F\left(t, y_{1}, y_{2}\right)\right\| \\
& \quad \leq L\left(\left\|x_{1}-y_{1}\right\|+\left\|x_{2}-y_{2}\right\|\right)
\end{aligned}
$$

uniformly in $t \in \mathbb{R}$, where $L>0$ is called the Lipschitz constant for the function $F(t, x, y)$, then $F(t, x, y)$ is uniformly continuous on $K$ uniformly for $t \in \mathbb{R}$, where $K$ is any bounded subset of $X \times X$. 
Remark 8. If $F(t, x, y)$ satisfies a local Lipschitz condition with respect to $x$ and $y$ uniformly in $t \in \mathbb{R}$, i.e., for each pair $x_{1}, x_{2}, y_{1}, y_{2} \in X, t \in \mathbb{R}$,

$$
\begin{aligned}
& \left\|F\left(t, x_{1}, x_{2}\right)-F\left(t, y_{1}, y_{2}\right)\right\| \\
& \quad \leq L(t)\left(\left\|x_{1}-y_{1}\right\|+\left\|x_{2}-y_{2}\right\|\right),
\end{aligned}
$$

where $L(t) \in B C\left(\mathbb{R}, \mathbb{R}^{+}\right)$, then $F(t, x, y)$ is uniformly continuous on $K$ uniformly for $t \in \mathbb{R}$, where $K$ is any bounded subset of $X \times X$.

Definition 9 (see [6]). A continuous function $F: \mathbb{R} \rightarrow X$ is said to be asymptotically almost automorphic if it can be decomposed as $F(t)=G(t)+\Phi(t)$, where

$$
\begin{aligned}
& G(t) \in A A(\mathbb{R}, X), \\
& \Phi(t) \in C_{0}(\mathbb{R}, X) .
\end{aligned}
$$

Denote by $A A A(\mathbb{R}, X)$ the set of all such functions.

Remark 10. The function $F: \mathbb{R} \longrightarrow \mathbb{R}$ defined by

$$
\begin{aligned}
F(t) & =G(t)+\Phi(t) \\
& =\sin \left(\frac{1}{2+\cos t+\cos \sqrt{2} t}\right)+e^{-|t|}
\end{aligned}
$$

is an asymptotically almost automorphic function with

$$
\begin{aligned}
& G(t)=\sin \left(\frac{1}{2+\cos t+\cos \sqrt{2} t}\right) \in A A(\mathbb{R}, \mathbb{R}), \\
& \Phi(t)=e^{-|t|} \in C_{0}(\mathbb{R}, \mathbb{R}) .
\end{aligned}
$$

Lemma 11 (see [6]). AAA( $\mathbb{R}, X)$ is also a Banach space with the supremum norm $\|\cdot\|_{\infty}$.

Definition 12 (see [6]). A continuous function $F: \mathbb{R} \times Y \times$ $Y \longrightarrow X$ is said to be asymptotically almost automorphic if it can be decomposed as $F(t, x, y)=G(t, x, y)+\Phi(t, x, y)$, where

$$
\begin{aligned}
& G(t, x, y) \in A A(\mathbb{R} \times Y \times Y, X), \\
& \Phi(t, x, y) \in C_{0}(\mathbb{R} \times Y \times Y, X) .
\end{aligned}
$$

Denote by $A A A(\mathbb{R} \times Y \times Y, X)$ the set of all such functions.

Remark 13. The function $F: \mathbb{R} \times X \times X \longrightarrow X$ given by

$$
\begin{aligned}
F(t, x, y)= & G(t, x, y)+\Phi(t, x, y) \\
= & \sin \left(\frac{1}{2+\cos t+\cos \sqrt{2} t}\right)[\sin (x)+y] \\
& +e^{-|t|}[x+\sin (y)]
\end{aligned}
$$

is asymptotically almost automorphic in $t \in \mathbb{R}$ uniformly for all $(x, y) \in K$, where $K$ is any bounded subset of $X \times X, X=$ $L^{2}[0, \pi]$ and

$$
\begin{aligned}
G(t, x, y) & =\sin \left(\frac{1}{2+\cos t+\cos \sqrt{2} t}\right)[\sin (x)+y] \\
& \in A A(\mathbb{R} \times X \times X, X), \\
\Phi(t, x, y) & =e^{-|t|}[x+\sin (y)] \in C_{0}(\mathbb{R} \times X \times X, X) .
\end{aligned}
$$

Next we give some basic definitions and properties of the fractional calculus theory which are used further in this paper.

Definition 14 (see [26]). The fractional integral of order $\alpha>0$ with the lower limit $t_{0}$ for a function $f$ is defined as

$$
I^{\alpha} f(t)=\frac{1}{\Gamma(\alpha)} \int_{t_{0}}^{t}(t-s)^{\alpha-1} f(s) \mathrm{d} s, \quad t>t_{0}, \quad \alpha>0
$$

provided that the right-hand side is point-wise defined on $\left[t_{0}, \infty\right)$, where $\Gamma$ is the Gamma function.

Definition 15 (see [26]). Riemann-Liouville derivative of order $\alpha>0$ with the lower limit $t_{0}$ for a function $f$ : $\left[t_{0}, \infty\right) \longrightarrow \mathbb{R}$ can be written as

$$
\begin{aligned}
& D_{t}^{\alpha} f(t)=\frac{1}{\Gamma(n-\alpha)} \frac{\mathrm{d}^{n}}{\mathrm{~d} t^{n}} \int_{t_{0}}^{t}(t-s)^{-\alpha} f(s) \mathrm{d} s, \\
& t>t_{0}, n-1<\alpha<n .
\end{aligned}
$$

The first and maybe the most important property of Riemann-Liouville fractional derivative is that, for $t>t_{0}$ and $\alpha>0$, one has $D_{t}^{\alpha}\left(I^{\alpha} f(t)\right)=f(t)$, which means that Riemann-Liouville fractional differentiation operator is a left inverse to the Riemann-Liouville fractional integration operator of the same order $\alpha$.

It is important to define sectorial operator for the definition of mild solution of any fractional abstract equations. So, let us now give the definitions of sectorial linear operators and their associated solution operators.

Definition 16 ([74] sectorial operator). A closed and linear operator $A$ is said to be sectorial of type $\omega$ and angle $\theta$ if there exist $0<\theta<\pi / 2, M>0$, and $\omega \in \mathbb{R}$ such that its resolvent $\rho(A)$ exists outside the sector $\omega+S_{\theta}:=\{\omega+\lambda: \lambda \epsilon$ $\mathbb{C},|\arg (-\lambda)|<\theta\}$ and

$$
\left\|(\lambda-A)^{-1}\right\| \leq \frac{M}{|\lambda-\omega|}, \quad \lambda \notin \omega+S_{\theta} .
$$

Sectorial operators are well studied in the literature, usually for the case $\omega=0$. For a recent reference including several examples and properties we refer the reader to [74]. Note that an operator $A$ is sectorial of type $\omega$ if and only if $\omega I-A$ is sectorial of type 0 .

Definition 17 (see [75]). Let $A$ be a closed and linear operator with domain $D(A)$ defined on a Banach space $X$. We call $A$ 
the generator of a solution operator if there are $\omega \in \mathbb{R}$ and a strongly continuous function $S_{\alpha}: \mathbb{R}^{+} \longrightarrow \mathbb{R}(X)$ such that $\left\{\lambda^{\alpha}: \operatorname{Re} \lambda>\omega\right\} \subseteq \rho(A)$ and

$$
\begin{array}{r}
\lambda^{\alpha-1}\left(\lambda^{\alpha}-A\right)^{-1} x=\int_{0}^{\infty} e^{-\lambda t} S_{\alpha}(t) x \mathrm{~d} t, \\
\operatorname{Re} \lambda>\omega, x \in X .
\end{array}
$$

In this case, $S_{\alpha}(t)$ is called the solution operator generated by A.

Note that if $A$ is sectorial of type $\omega$ with $0 \leq \theta \leq \pi(1-\alpha / 2)$, then $A$ is the generator of a solution operator given by

$$
S_{\alpha}(t):=\frac{1}{2 \pi i} \int_{\gamma} e^{-\lambda t} \lambda^{\alpha-1}\left(\lambda^{\alpha}-A\right)^{-1} \mathrm{~d} \lambda,
$$

where $\gamma$ is a suitable path lying outside the sector $\omega+\Sigma_{\theta}$ (cf. [74]).

Very recently, Cuesta in [74](Theorem 1) has proved that if $A$ is a sectorial operator of type $\omega<0$ for some $M>0$ and $0 \leq \theta<\pi(1-\alpha / 2)$, then there exists $C>0$ such that

$$
\left\|S_{\alpha}(t)\right\|_{\mathbb{L}(X)} \leq \frac{C M}{1+|\omega| t^{\alpha}} \text { for } t \geq 0 .
$$

In the border case $\alpha=1$, this is analogous to saying that $A$ is the generator of a exponentially stable $C_{0}$-semigroup. The main difference is that in the case $\alpha>1$ the solution family $S_{\alpha}(t)$ decays like $t^{-\alpha}$. Cuesta's result proves that $S_{\alpha}(t)$ is, in fact, integrable.

In the following, we present the following compactness criterion, which is a special case of the general compactness result of Theorem 2.1 in [76].

Lemma 18 (see [76]). A set $D \subset C_{0}(\mathbb{R}, X)$ is relatively compact if

(1) D is equicontinuous;

(2) $\lim _{|t| \rightarrow \infty} x(t)=0$ uniformly for $x \in D$;

(3) the set $D(t):=\{x(t): x \in D\}$ is relatively compact in $X$ for every $t \in \mathbb{R}$.

The following Krasnoselskii's fixed point theorem plays a key role in the proofs of our main results, which can be found in many books.

Lemma 19 (see [77]). Let $U$ be a bounded closed and convex subset of $X$ and $J_{1}, J_{2}$ be maps of $U$ into $X$ such that $J_{1} x+J_{2} y \in$ $U$ for every pair $x, y \in U$. If $J_{1}$ is a contraction and $J_{2}$ is completely continuous, then $J_{1} x+J_{2} x=x$ has a solution on $U$.

\section{Asymptotically Almost Automorphic Mild Solutions}

In this section, we study the existence of asymptotically almost automorphic mild solutions for the semilinear fractional differential equations of the form

$$
\begin{aligned}
\mathrm{D}_{t}^{\alpha} x(t)=A x(t)+\mathrm{D}_{t}^{\alpha-1} F(t, x(t), & B x(t)), \\
& t \in \mathbb{R}, 1<\alpha<2,
\end{aligned}
$$

where $A: D(A) \subset X \longrightarrow X$ is a linear densely defined operator of sectorial type of $\omega<0$ on a complex Banach space $X, B: X \longrightarrow X$ is a bounded linear operator and $F: \mathbb{R} \times X \times X \longrightarrow X$, and $(t, x, y) \longrightarrow F(t, x, y)$ is a given function to be specified later. The fractional derivative $D_{t}^{\alpha}$ is to be understood in Riemann-Liouville sense.

We recall the following definition that will be essential for us.

Definition 20 (see [63]). Assume that $A$ generates an integrable solution operator $S_{\alpha}(t)$. A continuous function $x$ : $\mathbb{R} \longrightarrow X$ satisfying the integral equation

$$
x(t)=\int_{-\infty}^{t} S_{\alpha}(t-\sigma) F(\sigma, x(\sigma), B x(\sigma)) \mathrm{d} \sigma, \quad t \in \mathbb{R}
$$

is called a mild solution on $\mathbb{R}$ to (39).

In the proofs of our results, we need the following auxiliary result.

Lemma 21. Given $Y(t) \in A A(\mathbb{R}, X)$ and $Z(t) \in C_{0}(\mathbb{R}, X)$, let

$$
\begin{aligned}
& \Phi_{1}(t):=\int_{-\infty}^{t} S_{\alpha}(t-s) Y(s) d s \\
& \Phi_{2}(t):=\int_{-\infty}^{t} S_{\alpha}(t-s) Z(s) d s
\end{aligned}
$$

$t \in \mathbb{R}$.

Then $\Phi_{1}(t) \in A A(\mathbb{R}, X), \Phi_{2}(t) \in C_{0}(\mathbb{R}, X)$.

Proof. Firstly, note that

$$
\int_{0}^{\infty} \frac{1}{1+|\omega| s^{\alpha}} \mathrm{d} s=\frac{|\omega|^{-1 / \alpha} \pi}{\alpha \sin (\pi / \alpha)} \quad \text { for } 1<\alpha<2 .
$$

Then

$$
\begin{aligned}
\left\|\Phi_{1}(t)\right\| & =\left\|\int_{-\infty}^{t} S_{\alpha}(t-s) Y(s) \mathrm{d} s\right\| \\
& =\left\|\int_{0}^{+\infty} S_{\alpha}(\tau) Y(t-\tau) \mathrm{d} \tau\right\| \\
& \leq C M\|Y\|_{\infty} \int_{0}^{\infty} \frac{1}{1+|\omega| \tau^{\alpha}} \mathrm{d} \tau \\
& =\frac{C M|\omega|^{-1 / \alpha} \pi}{\alpha \sin (\pi / \alpha)}\|Y\|_{\infty},
\end{aligned}
$$

which implies that $\Phi_{1}(t)$ is well defined and continuous on $\mathbb{R}$. Since $Y(t) \in A A(\mathbb{R}, X)$, then for any $\varepsilon>0$ and every sequence of real numbers $\left\{s_{n}^{\prime}\right\}$, there exist a subsequence $\left\{s_{n}\right\}$, a function $\tilde{Y}(t)$, and $N \in \mathbb{N}$ such that

$$
\left\|Y\left(s+s_{n}\right)-\tilde{Y}(s)\right\|<\varepsilon
$$

for each $n>N$ and every $s \in \mathbb{R}$. 
Define

$$
\widetilde{\Phi_{1}}(t):=\int_{-\infty}^{t} T(t-s) \widetilde{Y}(s) \mathrm{d} s
$$

Then

$$
\begin{aligned}
& \left\|\Phi_{1}\left(t+s_{n}\right)-\widetilde{\Phi_{1}}(t)\right\|=\| \int_{-\infty}^{t+s_{n}} S_{\alpha}\left(t+s_{n}-s\right) Y(s) \mathrm{d} s \\
& -\int_{-\infty}^{t} S_{\alpha}(t-s) Y(s) \mathrm{d} s \| \\
& =\| \int_{0}^{+\infty} S_{\alpha}(s) Y\left(t+s_{n}-s\right) \mathrm{d} s \\
& -\int_{0}^{+\infty} S_{\alpha}(s) Y(t-s) \mathrm{d} s \| \\
& \leq C M \int_{0}^{\infty} \frac{1}{1+|\omega| s^{\alpha}}\left\|Y\left(s+s_{n}\right)-\tilde{Y}(s)\right\| \mathrm{d} s \\
& \leq \frac{C M|\omega|^{-1 / \alpha} \pi \varepsilon}{\alpha \sin (\pi / \alpha)}
\end{aligned}
$$

for each $n>N$ and every $t \in \mathbb{R}$. This implies that

$$
\widetilde{\Phi_{1}}(t)=\lim _{n \longrightarrow \infty} \Phi_{1}\left(t+s_{n}\right)
$$

is well defined for each $t \in \mathbb{R}$.

By a similar argument one can obtain

$$
\lim _{n \longrightarrow \infty} \widetilde{\Phi_{1}}\left(t-s_{n}\right)=\Phi_{1}(t) \quad \text { for each } t \in \mathbb{R} .
$$

Thus $\Phi_{1}(t) \in A A(\mathbb{R}, X)$.

Since $Z(t) \in C_{0}(\mathbb{R}, X)$, one can choose an $N_{1}>0$ such that $\|Z(t)\|<\varepsilon$ for all $t>N_{1}$. This enables us to conclude that, for all $t>N_{1}$,

$$
\begin{aligned}
\left\|\Phi_{2}(t)\right\| \leq & \left\|\int_{-\infty}^{N_{1}} S_{\alpha}(t-s) Z(s) \mathrm{d} s\right\| \\
& +\left\|\int_{N_{1}}^{t} S_{\alpha}(t-s) Z(s) \mathrm{d} s\right\| \\
\leq & C M\|Z\|_{\infty} \int_{-\infty}^{N_{1}} \frac{1}{1+|\omega|(t-s)^{\alpha}} \mathrm{d} s \\
& +\varepsilon C M \int_{N_{1}}^{t} \frac{1}{1+|\omega|(t-s)^{\alpha}} \mathrm{d} s \\
\leq & \frac{C M\|Z\|_{\infty}}{|\omega|} \int_{-\infty}^{N_{1}} \frac{1}{(t-s)^{\alpha}} \mathrm{d} s \\
& +\frac{C M|\omega|^{-1 / \alpha} \pi \varepsilon}{\alpha \sin (\pi / \alpha)}
\end{aligned}
$$

$$
\begin{aligned}
\leq & \frac{C M\|Z\|_{\infty}}{|\omega|} \frac{1}{(\alpha-1)\left(t-N_{1}\right)^{\alpha-1}} \\
& +\frac{C M|\omega|^{-1 / \alpha} \pi \varepsilon}{\alpha \sin (\pi / \alpha)},
\end{aligned}
$$

which implies

$$
\lim _{t \longrightarrow+\infty}\left\|\Phi_{2}(t)\right\|=0 .
$$

On the other hand, from $Z(t) \in C_{0}(\mathbb{R}, X)$ it follows that there exists an $N_{2}>0$ such that $\|Z(t)\|<\varepsilon$ for all $t<-N_{2}$. This enables us to conclude that, for all $t<-N_{2}$,

$$
\begin{aligned}
\left\|\Phi_{2}(t)\right\| & =\left\|\int_{-\infty}^{t} S_{\alpha}(t-s) Z(s) \mathrm{d} s\right\| \\
& \leq \int_{-\infty}^{t}\left\|S_{\alpha}(t-s)\right\|\|Z(s)\| \mathrm{d} s \\
& \leq C M \varepsilon \int_{-\infty}^{t} \frac{1}{1+|\omega|(t-s)^{\alpha}} \mathrm{d} s \\
& =\frac{C M|\omega|^{-1 / \alpha} \pi \varepsilon}{\alpha \sin (\pi / \alpha)},
\end{aligned}
$$

which implies

$$
\lim _{t \rightarrow-\infty}\left\|\Phi_{2}(t)\right\|=0
$$

Now we are in position to state and prove our first main result. To prove our main result, let us introduce the following assumptions:

$\left(H_{1}\right) F(t, x, y)=F_{1}(t, x, y)+F_{2}(t, x, y) \in A A A(\mathbb{R} \times X \times$ $X, X)$ with

$$
\begin{aligned}
& F_{1}(t, x, y) \in A A(\mathbb{R} \times X \times X, X), \\
& F_{2}(t, x, y) \in C_{0}(\mathbb{R} \times X \times X, X)
\end{aligned}
$$

and there exists a constant $L>0$ such that, for all $t \in \mathbb{R}$ and $x_{1}, x_{2}, y_{1}, y_{2} \in X$,

$$
\begin{aligned}
& \left\|F_{1}\left(t, x_{1}, x_{2}\right)-F_{1}\left(t, y_{1}, y_{2}\right)\right\| \\
& \quad \leq L\left(\left\|x_{1}-y_{1}\right\|+\left\|x_{2}-y_{2}\right\|\right) .
\end{aligned}
$$

$\left(H_{2}\right)$ There exist a function $\beta(t) \in C_{0}\left(\mathbb{R}, \mathbb{R}^{+}\right)$and a nondecreasing function $\Phi: \mathbb{R}^{+} \longrightarrow \mathbb{R}^{+}$such that, for all $t \in \mathbb{R}$ and $x, y \in X$ with $\|x\|+\|y\| \leq r$,

$$
\begin{aligned}
\left\|F_{2}(t, x, y)\right\| & \leq \beta(t) \Phi(r) \\
\text { and } \liminf _{r \rightarrow+\infty} \frac{\Phi(r)}{r} & =\rho_{1} .
\end{aligned}
$$

Remark 22. Assuming that $F(t, x, y)$ satisfies the assumption $\left(H_{1}\right)$, it is noted that $F(t, x, y)$ does not have to meet the 
Lipschitz continuity with respect to $x$ and $y$. Such class of asymptotically almost automorphic functions $F(t, x, y)$ are more complicated than those with Lipschitz continuity with respect to $x$ and $y$ and little is known about them. Define

Let $\beta(t)$ be the function involved in assumption $\left(\mathrm{H}_{2}\right)$.

$$
\sigma(t):=\int_{-\infty}^{t} \frac{\beta(s)}{1+|\omega|(t-s)^{\alpha}} \mathrm{d} s, \quad t \in \mathbb{R} .
$$

Lemma 23. $\sigma(t) \in C_{0}\left(\mathbb{R}, \mathbb{R}^{+}\right)$.

Proof. Since $\beta(t) \in C_{0}\left(\mathbb{R}, \mathbb{R}^{+}\right)$, one can choose a $T_{1}>0$ such that $\|\beta(t)\|<\varepsilon$ for all $t>T_{1}$. This enables us to conclude that, for all $t>T_{1}$,

$$
\begin{aligned}
\|\sigma(t)\| \leq & \left\|\int_{-\infty}^{T_{1}} \frac{\beta(s)}{1+|\omega|(t-s)^{\alpha}} \mathrm{d} s\right\| \\
& +\left\|\int_{T_{1}}^{t} \frac{\beta(s)}{1+|\omega|(t-s)^{\alpha}} \mathrm{d} s\right\| \\
\leq & \|\beta\|_{\infty} \int_{-\infty}^{T_{1}} \frac{1}{1+|\omega|(t-s)^{\alpha}} \mathrm{d} s \\
& +\varepsilon \int_{T_{1}}^{t} \frac{1}{1+|\omega|(t-s)^{\alpha}} \mathrm{d} s \\
\leq & \frac{\|\beta\|_{\infty}}{|\omega|} \int_{-\infty}^{T_{1}} \frac{1}{(t-s)^{\alpha}} \mathrm{d} s+\frac{|\omega|^{-1 / \alpha} \pi \varepsilon}{\alpha \sin (\pi / \alpha)} \\
\leq & \frac{\|\beta\|_{\infty}}{|\omega|} \frac{1}{(\alpha-1)\left(t-T_{1}\right)^{\alpha-1}}+\frac{|\omega|^{-1 / \alpha} \pi \varepsilon}{\alpha \sin (\pi / \alpha)}
\end{aligned}
$$

which implies

$$
\lim _{t \longrightarrow+\infty}\|\sigma(t)\|=0 .
$$

On the other hand, from $\beta(t) \in C_{0}\left(\mathbb{R}, \mathbb{R}^{+}\right)$it follows that there exists a $T_{2}>0$ such that $\|\beta(t)\|<\varepsilon$ for all $t<-T_{2}$. This enables us to conclude that, for all $t<-T_{2}$,

$$
\begin{aligned}
\|\sigma(t)\| & =\left\|\int_{-\infty}^{t} \frac{\beta(s)}{1+|\omega|(t-s)^{\alpha}} \mathrm{d} s\right\| \\
& \leq \varepsilon \int_{-\infty}^{t} \frac{1}{1+|\omega|(t-s)^{\alpha}} \mathrm{d} s=\frac{|\omega|^{-1 / \alpha} \pi \varepsilon}{\alpha \sin (\pi / \alpha)},
\end{aligned}
$$

which implies

$$
\lim _{t \longrightarrow-\infty}\|\sigma(t)\|=0
$$

Theorem 24. Assume that $A$ is sectorial of type $\omega<0$. Let $F: \mathbb{R} \times X \times X \longrightarrow X$ satisfy the hypotheses $\left(H_{1}\right)$ and $\left(H_{2}\right)$. Put $\rho_{2}:=\sup _{t \in \mathbb{R}} \sigma(t)$. Then (39) has at least one asymptotically almost automorphic mild solution provided that

$$
\begin{aligned}
& \frac{C M L\left(1+\|B\|_{\mathbb{L}(X)}\right)|\omega|^{-1 / \alpha} \pi}{\alpha \sin (\pi / \alpha)} \\
& +C M\left(1+\|B\|_{\mathbb{L}(X)}\right) \rho_{1} \rho_{2}<1 .
\end{aligned}
$$

Proof. The proof is divided into the following five steps.

Step 1. Define a mapping $\Lambda$ on $A A(\mathbb{R}, X)$ by

$$
(\Lambda v)(t)=\int_{-\infty}^{t} S_{\alpha}(t-s) F_{1}(s, v(s), B v(s)) \mathrm{d} s
$$

$$
t \in \mathbb{R}
$$

and prove $\Lambda$ has a unique fixed point $v(t) \in A A(\mathbb{R}, X)$.

Firstly, since the function $s \longrightarrow F_{1}(s, v(s), B v(s))$ is bounded in $\mathbb{R}$ and

$$
\begin{aligned}
& \|[\Lambda v](t)\| \leq \int_{-\infty}^{t}\left\|S_{\alpha}(t-s)\right\|\left\|F_{1}(s, v(s), B v(s))\right\| \mathrm{d} s \\
& \leq C M \int_{-\infty}^{t} \frac{1}{1+|\omega|(t-s)^{\alpha}}\left\|F_{1}(s, v(s), B v(s))\right\| \mathrm{d} s \\
& \leq C M\left\|F_{1}\right\|_{\infty} \int_{-\infty}^{t} \frac{1}{1+|\omega|(t-s)^{\alpha}} \mathrm{d} s \\
& =\frac{C M L|\omega|^{-1 / \alpha} \pi\left\|F_{1}\right\|_{\infty}}{\alpha \sin (\pi / \alpha)},
\end{aligned}
$$

this implies that $(\Lambda v)(t)$ exists. Moreover from $F_{1}(t, x, y) \in$ $A A(\mathbb{R} \times X \times X, X)$ satisfying (54), together with Lemma 6 and Remark 7, it follows that

$$
F_{1}(\cdot, v(\cdot), B v(\cdot)) \in A A(\mathbb{R}, X)
$$

$$
\text { for every } v(\cdot) \in A A(\mathbb{R}, X) \text {. }
$$

This, together with Lemma 21, implies that $\Lambda$ is well defined and maps $A A(\mathbb{R}, X)$ into itself.

In the sequel, we verify that $\Lambda$ is continuous.

Let $v_{n}(t), v(t)$ be in $A A(\mathbb{R}, X)$ with $v_{n}(t) \longrightarrow v(t)$ as $n \longrightarrow$ $\infty$; then one has

$$
\begin{aligned}
& \left\|\left[\Lambda v_{n}\right](t)-[\Lambda v](t)\right\|=\| \int_{-\infty}^{t} S_{\alpha}(t-s) \\
& \cdot\left[F_{1}\left(s, v_{n}(s), B v_{n}(s)\right)\right. \\
& \left.-F_{1}(s, v(s), B v(s))\right] \mathrm{d} s\left\|\leq L \int_{-\infty}^{t}\right\| S_{\alpha}(t-s) \| \\
& \cdot\left[\left\|v_{n}(s)-v(s)\right\|+\left\|B v_{n}(s)-B v(s)\right\|\right] \mathrm{d} s \\
& \leq C M L \int_{-\infty}^{t} \frac{1}{1+|\omega|(t-s)^{\alpha}}\left(1+\|B\|_{\mathbb{L}(X)}\right) \| v_{n}(s) \\
& \quad-v(s)\left\|\mathrm{d} s \leq C M L\left(1+\|B\|_{\mathbb{L}(X)}\right)\right\| v_{n}-v \|_{\infty}
\end{aligned}
$$




$$
\begin{aligned}
& \cdot \int_{-\infty}^{t} \frac{1}{1+|\omega|(t-s)^{\alpha}} \mathrm{d} s \\
& =\frac{C M L\left(1+\|B\|_{\mathbb{L}(X)}\right)|\omega|^{-1 / \alpha} \pi}{\alpha \sin (\pi / \alpha)}\left\|v_{n}-v\right\|_{\infty} .
\end{aligned}
$$

Therefore, as $n \longrightarrow \infty$ and $\Lambda v_{n} \longrightarrow \Lambda v$, hence $\Lambda$ is continuous.

Next, we prove that $\Lambda$ is a contraction on $A A(\mathbb{R}, X)$ and has a unique fixed point $v(t) \in A A(\mathbb{R}, X)$.

In fact, let $v_{1}(t), v_{2}(t)$ be in $A A(\mathbb{R}, X)$, and similar to the above proof of the continuity of $\Lambda$, one has

$$
\begin{aligned}
& \left\|\left[\Lambda v_{1}\right](t)-\left[\Lambda v_{2}\right](t)\right\| \\
& \quad \leq \frac{C M L\left(1+\|B\|_{\mathbb{L}(X)}\right)|\omega|^{-1 / \alpha} \pi}{\alpha \sin (\pi / \alpha)}\left\|v_{1}-v_{2}\right\|_{\infty},
\end{aligned}
$$

which implies

$$
\begin{aligned}
& \left\|\left[\Lambda v_{1}\right](t)-\left[\Lambda v_{2}\right](t)\right\|_{\infty} \\
& \quad \leq \frac{C M L\left(1+\|B\|_{\mathbb{L}(X)}\right)|\omega|^{-1 / \alpha} \pi}{\alpha \sin (\pi / \alpha)}\left\|v_{1}-v_{2}\right\|_{\infty} .
\end{aligned}
$$

Together with (61), this proves that $\Lambda$ is a contraction on $A A(\mathbb{R}, X)$. Thus, Banach's fixed point theorem implies that $\Lambda$ has a unique fixed point $v(t) \in A A(\mathbb{R}, X)$.

Step 2. Set

$$
\Omega_{r}:=\left\{\omega(t) \in C_{0}(\mathbb{R}, X):\|\omega(t)\| \leq r\right\} .
$$

For the above $v(t)$, define $\Gamma:=\Gamma^{1}+\Gamma^{2}$ on $C_{0}(\mathbb{R}, X)$ as

$$
\begin{aligned}
& \left(\Gamma^{1} \omega\right)(t)=\int_{-\infty}^{t} S_{\alpha}(t-s) \\
& \cdot\left[F_{1}(s, v(s)+\omega(s), B(v(s)+\omega(s)))\right. \\
& \left.-F_{1}(s, v(s), B v(s))\right] \mathrm{d} s, \\
& \left(\Gamma^{2} \omega\right)(t)=\int_{-\infty}^{t} S_{\alpha}(t-s) F_{2}(s, v(s) \\
& +\omega(s), B(v(s)+\omega(s))) \mathrm{d} s
\end{aligned}
$$

and prove that $\Gamma$ maps $\Omega_{k_{0}}$ into itself, where $k_{0}$ is a given constant. $X$,

Firstly, from (54) it follows that, for all $s \in \mathbb{R}$ and $\omega(s) \in$

$$
\begin{aligned}
& \| F_{1}(s, v(s)+\omega(s), B(v(s)+\omega(s))) \\
& \quad-F_{1}(s, v(s), B v(s)) \| \leq L[\|\omega(s)\|+\|B \omega(s)\|] \\
& \quad \leq L\left(1+\|B\|_{\mathbb{L}(X)}\right)\|\omega(s)\|,
\end{aligned}
$$

which implies that

$$
\begin{gathered}
F_{1}(\cdot, v(\cdot)+\omega(\cdot), B(v(\cdot)+\omega(\cdot)))-F_{1}(\cdot, v(\cdot), B v(\cdot)) \\
\quad \in C_{0}(\mathbb{R}, X) \quad \text { for every } \omega(\cdot) \in C_{0}(\mathbb{R}, X) .
\end{gathered}
$$

According to (55), one has

$$
\begin{aligned}
& \left\|F_{2}(s, v(s)+\omega(s), B(v(s)+\omega(s)))\right\| \leq \beta(s) \\
& . \Phi\left(\|\omega(s)+B \omega(s)\|+\sup _{s \in \mathbb{R}}\|v(s)+B v(s)\|\right) \\
& \leq \beta(s) \Phi\left(\left(1+\|B\|_{\mathbb{L}(X)}\right)\|\omega(s)\|\right. \\
& \left.+\left(1+\|B\|_{\mathbb{L}(X)}\right) \sup _{s \in \mathbb{R}}\|v(s)\|\right)=\beta(s) \\
& \cdot \Phi\left(\left(1+\|B\|_{\mathbb{L}(X)}\right)\left[\|\omega(s)\|+\sup _{s \in \mathbb{R}}\|v(s)\|\right]\right)
\end{aligned}
$$

for all $s \in \mathbb{R}$ and $\omega(s) \in X$ with $\|\omega(s)\| \leq r$; then

$$
\begin{aligned}
F_{2}(\cdot, v(\cdot)+\omega(\cdot), B(v(\cdot)+\omega(\cdot))) & \in C_{0}(\mathbb{R}, X) \\
& \text { as } \beta(\cdot) \in C_{0}\left(\mathbb{R}, \mathbb{R}^{+}\right) .
\end{aligned}
$$

Those, together with Lemma 21, yield that $\Gamma$ is well defined and maps $C_{0}(\mathbb{R}, X)$ into itself.

On the other hand, in view of (55) and (61) it is not difficult to see that there exists a constant $k_{0}>0$ such that

$$
\begin{aligned}
& \frac{C M L\left(1+\|B\|_{\mathbb{L}(X)}\right)|\omega|^{-1 / \alpha} \pi}{\alpha \sin (\pi / \alpha)} k_{0} \\
& \quad+C M \rho_{2} \Phi\left(\left(1+\|B\|_{\mathbb{L}(X)}\right)\left(k_{0}+\sup _{s \in \mathbb{R}}\|v(s)\|\right)\right)
\end{aligned}
$$

$\leq k_{0}$.

This enables us to conclude that, for any $t \in \mathbb{R}$ and $\omega_{1}(t)$, $\omega_{2}(t) \in \Omega_{k_{0}}$,

$$
\begin{aligned}
& \left\|\left(\Gamma^{1} \omega_{1}\right)(t)+\left(\Gamma^{2} \omega_{2}\right)(t)\right\| \leq \| \int_{-\infty}^{t} S_{\alpha}(t-s) \\
& \cdot\left[F_{1}\left(s, v(s)+\omega_{1}(s), B\left(v(s)+\omega_{1}(s)\right)\right)\right. \\
& \left.-F_{1}(s, v(s), B v(s))\right] \mathrm{d} s\|+\| \int_{-\infty}^{t} S_{\alpha}(t-s) \\
& \cdot F_{2}\left(s, v(s)+\omega_{2}(s), B\left(v(s)+\omega_{2}(s)\right)\right) \mathrm{d} s \| \\
& \leq \int_{-\infty}^{t}\left\|S_{\alpha}(t-s)\right\| \| F_{1}(s, v(s) \\
& \left.+\omega_{1}(s), B\left(v(s)+\omega_{1}(s)\right)\right) \\
& -F_{1}(s, v(s), B v(s))\left\|\mathrm{d} s+\int_{-\infty}^{t}\right\| S_{\alpha}(t-s) \| \\
& \cdot\left\|F_{2}\left(s, v(s)+\omega_{2}(s), B\left(v(s)+\omega_{2}(s)\right)\right)\right\| \mathrm{d} s \\
& \leq C M \int_{-\infty}^{t} \frac{1}{1+|\omega|(t-s)^{\alpha}}\left[\left\|\omega_{1}(s)\right\|\right.
\end{aligned}
$$


$\left.+\left\|B \omega_{1}(s)\right\|\right] \mathrm{d} s$

$+C M \int_{-\infty}^{t} \frac{\beta(s)}{1+|\omega|(t-s)^{\alpha}} \Phi\left(\left\|\omega_{2}(s)\right\|+\left\|B \omega_{2}(s)\right\|\right.$

$+\|v(s)\|+\|B v(s)\|) \mathrm{d} s \leq C M L\left(1+\|B\|_{\mathbb{L}(X)}\right)$

$\cdot\left\|\omega_{1}\right\|_{\infty} \int_{-\infty}^{t} \frac{1}{1+|\omega|(t-s)^{\alpha}} \mathrm{d} s+C M \sigma(t) \Phi((1$

$\left.\left.+\|B\|_{\mathbb{L}(X)}\right)\left(\left\|\omega_{2}\right\|_{\infty}+\|v(s)\|_{\infty}\right)\right)$

$=\frac{C M L|\omega|^{-1 / \alpha} \pi\left(1+\|B\|_{\mathbb{L}(X)}\right)}{\alpha \sin (\pi / \alpha)}\|\omega\|_{\infty}$

$+C M \rho_{2} \Phi\left(\left(1+\|B\|_{\mathbb{L}(X)}\right)\left(\left\|\omega_{2}\right\|_{\infty}+\|v(s)\|_{\infty}\right)\right)$

$\leq \frac{C M L\left(1+\|B\|_{\mathbb{L}(X)}\right)|\omega|^{-1 / \alpha} \pi}{\alpha \sin (\pi / \alpha)} k_{0}+C M \rho_{2} \Phi((1$

$\left.\left.+\|B\|_{\mathbb{L}(X)}\right)\left(k_{0}+\|v(s)\|_{\infty}\right)\right) \leq k_{0}$, which implies that $\left(\Gamma^{1} \omega_{1}\right)(t)+\left(\Gamma^{2} \omega_{2}\right)(t) \in \Omega_{k_{0}}$. Thus $\Gamma$ maps $\Omega_{k_{0}}$ into itself.

Step 3. Show that $\Gamma^{1}$ is a contraction on $\Omega_{k_{0}}$.

In fact, for any $\omega_{1}(t), \omega_{2}(t) \in \Omega_{k_{0}}$ and $t \in \mathbb{R}$, from (54) it follows that

$$
\begin{aligned}
\| & {\left[F_{1}\left(s, v(s)+\omega_{1}(s), B\left(v(s)+\omega_{1}(s)\right)\right)\right.} \\
& \left.-F_{1}(s, v(s), B v(s))\right] \\
& -\left[F_{1}\left(s, v(s)+\omega_{2}(s), B\left(v(s)+\omega_{2}(s)\right)\right)\right. \\
& \left.-F_{1}(s, v(s), B v(s))\right] \| \leq L\left[\left\|\omega_{1}(s)-\omega_{2}(s)\right\|\right. \\
& \left.+\left\|B \omega_{1}(s)-B \omega_{2}(s)\right\|\right] \leq L\left(1+\|B\|_{\mathbb{L}(X)}\right) \| \omega_{1}(s) \\
& -\omega_{2}(s) \| .
\end{aligned}
$$

Thus

$$
\begin{aligned}
& \left\|\left(\Gamma^{1} \omega_{1}\right)(t)-\left(\Gamma^{1} \omega_{2}\right)(t)\right\|=\| \int_{-\infty}^{t} S_{\alpha}(t-s)\left[\left(F_{1}\left(s, v(s)+\omega_{1}(s), B\left(v(s)+\omega_{1}(s)\right)\right)-F_{1}(s, v(s), B v(s))\right)\right. \\
& \left.-\left(F_{1}\left(s, v(s)+\omega_{2}(s), B\left(v(s)+\omega_{2}(s)\right)\right)-F_{1}(s, v(s), B v(s))\right)\right] \mathrm{d} s\left\|\leq L \int_{-\infty}^{t}\right\| S_{\alpha}(t-s)\left\|\left(1+\|B\|_{\mathbb{L}(X)}\right)\right\| \omega_{1}(s) \\
& -\omega_{2}(s)\left\|\mathrm{d} s \leq C M L\left(1+\|B\|_{\mathbb{L}(X)}\right)\right\| \omega_{1}-\omega_{2}\left\|_{\infty} \int_{-\infty}^{t} \frac{1}{1+|\omega|(t-s)^{\alpha}} \mathrm{d} s=\frac{C M L\left(1+\|B\|_{\mathbb{L}(X)}\right)|\omega|^{-1 / \alpha} \pi}{\alpha \sin (\pi / \alpha)}\right\| \omega_{1} \\
& -\omega_{2} \|_{\infty},
\end{aligned}
$$

which implies that

$$
\begin{aligned}
& \left\|\left(\Gamma^{1} \omega_{1}\right)(t)-\left(\Gamma^{1} \omega_{2}\right)(t)\right\|_{\infty} \\
& \quad \leq \frac{C M L\left(1+\|B\|_{\mathbb{L}(X)}\right)|\omega|^{-1 / \alpha} \pi}{\alpha \sin (\pi / \alpha)}\left\|\omega_{1}-\omega_{2}\right\|_{\infty} .
\end{aligned}
$$

Thus, in view of (61), one obtains the conclusion.

Step 4. Show that $\Gamma^{2}$ is completely continuous on $\Omega_{k_{0}}$.

Given $\varepsilon>0$. Let $\left\{\omega_{k}\right\}_{k=1}^{+\infty} \subset \Omega_{k_{0}}$ with $\omega_{k} \longrightarrow \omega_{0}$ in $C_{0}(\mathbb{R}, X)$ as $k \longrightarrow+\infty$. Since $\sigma(t) \in C_{0}\left(\mathbb{R}, \mathbb{R}^{+}\right)$, one may choose a $t_{1}>0$ big enough such that, for all $t \geq t_{1}$,

$$
\Phi\left(\left(1+\|B\|_{\mathbb{L}(X)}\right)\left(k_{0}+\|v\|_{\infty}\right)\right) \sigma(t)<\frac{\varepsilon}{3 C M} .
$$

Also, in view of $\left(H_{1}\right)$, we have

$$
\begin{aligned}
& F_{2}\left(s, v(s)+\omega_{k}(s), B\left(v(s)+\omega_{k}(s)\right)\right) \\
& \quad \longrightarrow F_{2}\left(s, v(s)+\omega_{0}(s), B\left(v(s)+\omega_{0}(s)\right)\right)
\end{aligned}
$$

for all $s \in\left(-\infty, t_{1}\right]$ as $k \longrightarrow+\infty$ and

$$
\begin{aligned}
& \| F_{2}\left(\cdot, v(\cdot)+\omega_{k}(\cdot), B\left(v(\cdot)+\omega_{k}(\cdot)\right)\right) \\
& \quad-F_{2}\left(\cdot, v(\cdot)+\omega_{0}(\cdot), B\left(v(\cdot)+\omega_{0}(\cdot)\right)\right) \| \\
& \quad \leq 2 \Phi\left(\left(1+\|B\|_{\mathbb{L}(X)}\right)\left(k_{0}+\|v\|_{\infty}\right)\right) \beta(\cdot) \\
& \quad \in L^{1}\left(-\infty, t_{1}\right] .
\end{aligned}
$$

Hence, by the Lebesgue dominated convergence theorem we deduce that there exists an $N>0$ such that

$$
\begin{gathered}
C M \int_{-\infty}^{t_{1}} \frac{1}{1+|\omega|(t-s)^{\alpha}} \| F_{2}(s, v(s) \\
\left.\quad+\omega_{k}(s), B\left(v(s)+\omega_{k}(s)\right)\right)-F_{2}(s, v(s) \\
\left.\quad+\omega_{0}(s), B\left(v(s)+\omega_{0}(s)\right)\right) \| \mathrm{d} s \leq \frac{\varepsilon}{3}
\end{gathered}
$$


whenever $k \geq N$. Thus

$$
\begin{aligned}
& \left\|\left(\Gamma^{2} \omega_{k}\right)(t)-\left(\Gamma^{2} \omega_{0}\right)(t)\right\|=\| \int_{-\infty}^{t} S_{\alpha}(t-s) F_{2}(s, v(s) \\
& \left.+\omega_{k}(s), B\left(v(s)+\omega_{k}(s)\right)\right) \mathrm{d} s-\int_{-\infty}^{t} S_{\alpha}(t \\
& \quad-s) F_{2}\left(s, v(s)+\omega_{0}(s), B\left(v(s)+\omega_{0}(s)\right)\right) \mathrm{d} s \| \\
& \quad \leq C M \int_{-\infty}^{t_{1}} \frac{1}{1+|\omega|(t-s)^{\alpha}} \| F_{2}(s, v(s) \\
& \left.+\omega_{k}(s), B\left(v(s)+\omega_{k}(s)\right)\right)-F_{2}(s, v(s) \\
& \left.+\omega_{0}(s), B\left(v(s)+\omega_{0}(s)\right)\right) \| \mathrm{d} s+2 C M \Phi((1 \\
& \left.\left.+\|B\|_{\mathbb{L}(X)}\right)\left(k_{0}+\|v\|_{\infty}\right)\right) \int_{t_{1}}^{\max \left\{t, t_{1}\right\}} \frac{\beta(s)}{1+|\omega|(t-s)^{\alpha}} \mathrm{d} s \\
& \quad \leq C M \int_{-\infty}^{t_{1}} \frac{1}{1+|\omega|(t-s)^{\alpha}} \| F_{2}(s, v(s) \\
& \left.\left.+\|B\|_{\mathbb{L}(X)}\right)\left(k_{0}+\|v\|_{\infty}\right)\right) \sigma(t) \leq \frac{\varepsilon}{3}+\frac{2 \varepsilon}{3}=\varepsilon \\
& \left.+\omega_{k}(s), B\left(v(s)+\omega_{k}(s)\right)\right)-F_{2}(s, v(s) \\
& \left.+\omega_{0}(s), B\left(v(s)+\omega_{0}(s)\right)\right) \| \mathrm{d} s+2 C M \Phi((1
\end{aligned}
$$

whenever $k \geq N$. Accordingly, $\Gamma^{2}$ is continuous on $\Omega_{k_{0}}$.

In the sequel, we consider the compactness of $\Gamma^{2}$.

Set $B_{r}(X)$ for the closed ball with center at 0 and radius $r$ in $X, V=\Gamma^{2}\left(\Omega_{k_{0}}\right)$, and $z(t)=\Gamma^{2}(u(t))$ for $u(t) \in \Omega_{k_{0}}$. First, for all $\omega(t) \in \Omega_{k_{0}}$ and $t \in \mathbb{R}$,

$$
\begin{aligned}
& \left\|\left(\Gamma^{2} \omega\right)(t)\right\|=\| \int_{-\infty}^{t} S_{\alpha}(t-s) \\
& \cdot F_{2}(s, v(s)+\omega(s), B(v(s)+\omega(s))) \mathrm{d} s \| \\
& \quad \leq C M \sigma(t) \Phi\left(\left(1+\|B\|_{\mathbb{L}(X)}\right)\left(k_{0}+\|v\|_{\infty}\right)\right),
\end{aligned}
$$

and in view of $\sigma(t) \in C_{0}\left(\mathbb{R}, \mathbb{R}^{+}\right)$, which follows from Lemma 23, one concludes that

$$
\lim _{|t| \longrightarrow+\infty}\left(\Gamma^{2} \omega\right)(t)=0 \quad \text { uniformly for } \omega(t) \in \Omega_{k_{0}} .
$$

As

$$
\begin{aligned}
& \left\|\left(\Gamma^{2} \omega\right)(t)\right\|=\| \int_{-\infty}^{t} S_{\alpha}(t-s) F_{2}(s, v(s) \\
& +\omega(s), B(v(s)+\omega(s))) \mathrm{d} s\|=\| \int_{0}^{+\infty} S_{\alpha}(\tau) \\
& \cdot F_{2}(t-\tau, v(t-\tau) \\
& +\omega(t-\tau), B(v(t-\tau)+\omega(t-\tau))) \mathrm{d} \tau \| .
\end{aligned}
$$

Hence, given $\varepsilon_{0}>0$, one can choose a $\xi>0$ such that

$$
\begin{aligned}
& \| \int_{\xi}^{+\infty} S_{\alpha}(\tau) F_{2}(t-\tau, v(t-\tau) \\
& \quad+\omega(t-\tau), B(v(t-\tau)+\omega(t-\tau))) \mathrm{d} \tau \| \\
& \quad<\varepsilon_{0} .
\end{aligned}
$$

Thus we get

$$
z(t) \in \overline{\xi c\left(\left\{S_{\alpha}(\tau) F_{2}(\lambda, v(\lambda)+\omega(\lambda), B(v(\lambda)+\omega(\lambda))): 0 \leq \tau \leq \xi, t-\xi \leq \lambda \leq \xi,\|\omega\|_{\infty} \leq r\right\}\right)}+B_{\varepsilon_{0}}(X),
$$

where $c(K)$ denotes the convex hull of $K$. Using that $S_{\alpha}(\cdot)$ is strongly continuous, we infer that

$$
\begin{aligned}
K & =\left\{S_{\alpha}(\tau) F_{2}(\lambda, v(\lambda)+\omega(\lambda), B(v(\lambda)+\omega(\lambda))): 0\right. \\
& \left.\leq \tau \leq \xi, t-\xi \leq \lambda \leq \xi,\|\omega\|_{\infty} \leq r\right\}
\end{aligned}
$$

is a relatively compact set and $V \subset \overline{\xi(K)}+B_{\varepsilon_{0}}(X)$, which implies that $V$ is a relatively compact subset of $X$.

Next, we verify the equicontinuity of the set $\left\{\left(\Gamma^{2} \omega\right)(t)\right.$ : $\left.\omega(t) \in \Omega_{k_{0}}\right\}$.

Let $k>0$ be small enough and $t_{1}, t_{2} \in \mathbb{R}$ and $\omega(t) \in \Omega_{k_{0}}$. Then by (55) we have

$$
\begin{gathered}
\left\|\left(\Gamma^{2} \omega\right)\left(t_{2}\right)-\left(\Gamma^{2} \omega\right)\left(t_{1}\right)\right\| \leq \int_{t_{1}}^{t_{2}} \| S_{\alpha}\left(t_{2}-s\right) \\
\cdot F_{2}(s, v(s)+\omega(s), B(v(s)+\omega(s))) \| \mathrm{d} s
\end{gathered}
$$

$$
\begin{aligned}
& +\int_{-\infty}^{t_{1}-k} \|\left[S_{\alpha}\left(t_{2}-s\right)-S_{\alpha}\left(t_{1}-s\right)\right] \\
& \cdot F_{2}(s, v(s)+\omega(s), B(v(s)+\omega(s))) \| \mathrm{d} s \\
& +\int_{t_{1}-k}^{t_{1}} \|\left[S_{\alpha}\left(t_{2}-s\right)-S_{\alpha}\left(t_{1}-s\right)\right] \\
& \cdot F_{2}(s, v(s)+\omega(s), B(v(s)+\omega(s))) \| \mathrm{d} s \\
& \leq C M \Phi\left(\left(1+\|B\|_{\mathbb{L}(X)}\right)\left(k_{0}+\|v\|_{\infty}\right)\right) \\
& \cdot \int_{t_{1}}^{t_{2}} \frac{\beta(s)}{1+|\omega|\left(t_{2}-s\right)^{\alpha}} \mathrm{d} s+\Phi\left(( 1 + \| B \| _ { \mathbb { L } ( X ) } ) \left(k_{0}\right.\right. \\
& \left.\left.+\|v\|_{\infty}\right)\right) \sup _{s \in\left[-\infty, t_{1}-k\right]}\left\|S_{\alpha}\left(t_{2}-s\right)-S_{\alpha}\left(t_{1}-s\right)\right\| \\
& \cdot \int_{-\infty}^{t_{1}-k} \beta(s) \mathrm{d} s+C M \Phi\left(( 1 + \| B \| _ { \mathbb { L } ( X ) } ) \left(k_{0}\right.\right.
\end{aligned}
$$




$$
\begin{aligned}
& \left.\left.+\|v\|_{\infty}\right)\right) \int_{t_{1}-k}^{t_{1}}\left(\frac{\beta(s)}{1+|\omega|\left(t_{2}-s\right)^{\alpha}}\right. \\
& \left.+\frac{\beta(s)}{1+|\omega|\left(t_{1}-s\right)^{\alpha}}\right) \mathrm{d} s \longrightarrow 0
\end{aligned}
$$$$
\text { as } t_{2}-t_{1} \longrightarrow 0, k \longrightarrow 0 \text {, }
$$

which implies the equicontinuity of the set $\left\{\left(\Gamma^{2} \omega\right)(t): \omega(t) \epsilon\right.$ $\left.\Omega_{k_{0}}\right\}$.

Now an application of Lemma 18 justifies the compactness of $\Gamma^{2}$.

Step 5. Show that (39) has at least one asymptotically almost automorphic mild solution.

Firstly, the complete continuity of $\Gamma^{2}$, together with the results of Steps 2 and 3 as well as Lemma 19, yields that $\Gamma$ has at least one fixed point $\omega(t) \in \Omega_{k_{0}}$; furthermore $\omega(t) \epsilon$ $C_{0}(\mathbb{R}, X)$.

Then, consider the following coupled system of integral equations:

$$
\begin{aligned}
& v(t)=\int_{-\infty}^{t} S_{\alpha}(t-s) F_{1}(s, v(s), B v(s)) \mathrm{d} s, \quad t \in \mathbb{R}, \\
& \omega(t)=\int_{-\infty}^{t} S_{\alpha}(t-s) \\
& \cdot\left[F_{1}(s, v(s)+\omega(s), B(v(s)+\omega(s)))\right. \\
& \left.-F_{1}(s, v(s), B v(s))\right] \mathrm{d} s+\int_{-\infty}^{t} S_{\alpha}(t-s) \\
& \cdot F_{2}(s, v(s)+\omega(s), B(v(s)+\omega(s))) \mathrm{d} s,
\end{aligned}
$$

$$
t \in \mathbb{R} \text {. }
$$

From the result of Step 1, together with the above fixed point $\omega(t) \in C_{0}(\mathbb{R}, X)$, it follows that

$$
(v(t), \omega(t)) \in A A(\mathbb{R}, X) \times C_{0}(\mathbb{R}, X)
$$

is a solution to system (91). Thus

$$
x(t):=v(t)+\omega(t) \in A A A(\mathbb{R}, X)
$$

and it is a solution to the integral equation

$$
x(t)=\int_{-\infty}^{t} S_{\alpha}(t-s) F(s, x(s), B x(s)) \mathrm{d} s, \quad t \in \mathbb{R} ;
$$

that is, $x(t)$ is an asymptotically almost automorphic mild solution to (39).

Taking $A=-\rho^{\alpha} I$ with $\rho>0$ in (39), the above theorem gives the following corollary.

Corollary 25. Let $F: \mathbb{R} \times X \times X \longrightarrow X$ satisfy $\left(H_{1}\right)$ and $\left(H_{2}\right)$. Put $\rho_{2}:=\sup _{t \in \mathbb{R}} \sigma(t)$. Then (39) admits at least one asymptotically almost automorphic mild solution whenever

$$
\frac{C L\left(1+\|B\|_{\mathbb{L}(X)}\right) \rho \pi}{\alpha \sin (\pi / \alpha)}+C\left(1+\|B\|_{\mathbb{L}(X)}\right) \rho_{1} \rho_{2}<1 .
$$

Remark 26. It is interesting to note that the function $\alpha \longrightarrow$ $\alpha \sin (\pi / \alpha) / \rho \pi$ is increasing from 0 to $2 / \rho \pi$ in the interval $1<$ $\alpha<2$. Therefore, with respect to condition (61), the class of admissible terms $F_{1}(t, x(t), B x(t))$ is the best in the case $\alpha=2$ and the worst in the case $\alpha=1$.

Theorem 24 can be extended to the case of $F_{1}(t, x, y)$ being locally Lipschitz continuous with respect to $x$ and $y$, where we have the following result.

$\left(H_{1}^{\prime}\right) F(t, x, y)=F_{1}(t, x, y)+F_{2}(t, x, y) \in A A A(\mathbb{R} \times X \times$ $X, X)$ with

$$
\begin{aligned}
& F_{1}(t, x, y) \in A A(\mathbb{R} \times X \times X, X), \\
& F_{2}(t, x, y) \in C_{0}(\mathbb{R} \times X \times X, X)
\end{aligned}
$$

and for all $x_{1}, x_{2}, y_{1}, y_{2} \in X, t \in \mathbb{R}$,

$$
\begin{aligned}
& \left\|F_{1}\left(t, x_{1}, x_{2}\right)-F_{1}\left(t, y_{1}, y_{2}\right)\right\| \\
& \quad \leq L(t)\left(\left\|x_{1}-y_{1}\right\|+\left\|x_{2}-y_{2}\right\|\right),
\end{aligned}
$$

where $L(t)$ is a function on $\mathbb{R}$.

Theorem 27. Assume that $A$ is sectorial of type $\omega<0$. Let $F: \mathbb{R} \times X \times X \longrightarrow X$ satisfy the hypotheses $\left(H_{1}^{\prime}\right)$ and $\left(H_{2}\right)$ with $L(t) \in B C\left(\mathbb{R}, \mathbb{R}^{+}\right)$. Put $\rho_{2}:=\sup _{t \in \mathbb{R}} \sigma(t)$. Let $\|L\|=$ $\sup _{t \in \mathbb{R}} \int_{t}^{t+1} L(s) \mathrm{d} s$. Then (39) has at least one asymptotically almost automorphic mild solution provided that

$$
\begin{gathered}
\frac{C M L\|L\||\omega|^{-1 / \alpha} \pi\left(1+\|B\|_{\mathbb{L}(X)}\right)}{\alpha \sin (\pi / \alpha)} \\
+C M \rho_{1} \rho_{2}\left(1+\|B\|_{\mathbb{L}(X)}\right)<1 .
\end{gathered}
$$

Proof. The proof is divided into the following five steps.

Step 1. Define a mapping $\Lambda$ on $A A(\mathbb{R}, X)$ by (62) and prove that $\Lambda$ has a unique fixed point $v(t) \in A A(\mathbb{R}, X)$.

Firstly, similar to the proof in Step 1 of Theorem 24, we can prove that $(\Lambda v)(t)$ exists. Moreover from $F_{1}(t, x, y) \epsilon$ $A A(\mathbb{R} \times X \times X, X)$ satisfying (97), together with Lemma 6 and Remark 8 , it follows that

$$
\begin{aligned}
F_{1}(\cdot, v(\cdot), B v(\cdot)) \in A A(\mathbb{R}, X) & \\
& \text { for every } v(\cdot) \in A A(\mathbb{R}, X) .
\end{aligned}
$$

This, together with Lemma 21, implies that $\Lambda$ is well defined and maps $A P(\mathbb{R}, X)$ into itself.

In the sequel, we verify that $\Lambda$ is continuous.

Let $v_{n}(t), v(t)$ be in $A A(\mathbb{R}, X)$ with $v_{n}(t) \longrightarrow v(t)$ as $n \longrightarrow$ $\infty$; then one has

$$
\begin{aligned}
& \left\|\left[\Lambda v_{n}\right](t)-[\Lambda v](t)\right\|=\| \int_{-\infty}^{t} S_{\alpha}(t-s) \\
& \cdot\left[F_{1}\left(s, v_{n}(s), B v_{n}(s)\right)\right. \\
& \left.-F_{1}(s, v(s), B v(s))\right] \mathrm{d} s\left\|\leq \int_{-\infty}^{t} L(s)\right\| S_{\alpha}(t \\
& -s) \|\left[\left\|v_{n}(s)-v(s)\right\|+\left\|B v_{n}(s)-B v(s)\right\|\right] \mathrm{d} s
\end{aligned}
$$




$$
\begin{aligned}
& \leq C M \int_{-\infty}^{t} \frac{L(s)}{1+|\omega|(t-s)^{\alpha}}\left(1+\|B\|_{\mathbb{L}(X)}\right) \| v_{n}(s) \\
& -v(s) \| \mathrm{d} s \leq C M\left(1+\|B\|_{\mathbb{L}(X)}\right) \\
& \cdot\left(\sum_{m=0}^{+\infty} \int_{t-(m+1)}^{t-m} \frac{L(s)}{1+|\omega|(t-s)^{\alpha}} \mathrm{d} s\right)\left\|v_{n}-v\right\|_{\infty} \\
& \leq C M\left(1+\|B\|_{\mathbb{L}(X)}\right) \\
& \cdot\left(\sum_{m=0}^{+\infty} \frac{1}{1+|\omega| m^{\alpha}} \int_{t-(m+1)}^{t-m} L(s) \mathrm{d} s\right)\left\|v_{n}-v\right\|_{\infty} \\
& \leq \frac{C M\|L\||\omega|^{-1 / \alpha} \pi\left(1+\|B\|_{\mathbb{L}(X)}\right)}{\alpha \sin (\pi / \alpha)}\left\|v_{n}-v\right\|_{\infty} .
\end{aligned}
$$

Therefore, as $n \longrightarrow \infty$ and $\Lambda v_{n} \longrightarrow \Lambda v$, hence $\Lambda$ is continuous.

Next, we prove that $\Lambda$ is a contraction on $A A(\mathbb{R}, X)$ and has a unique fixed point $v(t) \in A A(\mathbb{R}, X)$.

In fact, for $v_{1}(t), v_{2}(t)$ in $A A(\mathbb{R}, X)$, similar to the above proof of the continuity of $\Lambda$, one has

$$
\begin{aligned}
& \left\|\left(\Lambda v_{1}\right)(t)-\left(\Lambda v_{2}\right)(t)\right\| \\
& \quad \leq \frac{C M\|L\||\omega|^{-1 / \alpha} \pi\left(1+\|B\|_{\mathbb{L}(X)}\right)}{\alpha \sin (\pi / \alpha)}\left\|v_{1}-v_{2}\right\|_{\infty},
\end{aligned}
$$

which implies that

$$
\begin{aligned}
& \left\|\left(\Lambda v_{1}\right)(t)-\left(\Lambda v_{2}\right)(t)\right\|_{\infty} \\
& \quad \leq \frac{C M\|L\||\omega|^{-1 / \alpha} \pi\left(1+\|B\|_{\mathbb{L}(X)}\right)}{\alpha \sin (\pi / \alpha)}\left\|v_{1}-v_{2}\right\|_{\infty} .
\end{aligned}
$$

Hence, by (98), together with the contraction principle, $\Lambda$ has a unique fixed point $v(t) \in A A(\mathbb{R}, X)$.

Step 2. Set

$$
\Omega_{r}:=\left\{\omega(t) \in C_{0}(\mathbb{R}, X):\|\omega(t)\| \leq r\right\} .
$$

For the above $v(t)$, define $\Gamma:=\Gamma^{1}+\Gamma^{2}$ on $C_{0}(\mathbb{R}, X)$ as $(69)$ and prove that $\Gamma$ maps $\Omega_{k_{0}}$ into itself, where $k_{0}$ is a given constant.

Firstly, from (97) it follows that, for all $s \in \mathbb{R}, \omega(s) \in X$,

$$
\begin{aligned}
& \| F_{1}(s, v(s)+\omega(s), B(v(s)+\omega(s))) \\
& \quad-F_{1}(s, v(s), B v(s)) \| \leq L(s)[\|\omega(s)\|+\|B \omega(s)\|] \\
& \quad \leq L(s)\left(1+\|B\|_{\mathbb{L}(X)}\right)\|\omega(s)\|
\end{aligned}
$$

which together with $L(s) \in B C\left(\mathbb{R}, \mathbb{R}^{+}\right)$implies that

$$
\begin{aligned}
& F_{1}(\cdot, v(\cdot)+\omega(\cdot), B(v(\cdot)+\omega(\cdot))) \\
& \quad-F_{1}(\cdot, v(\cdot), B v(\cdot)) \in C_{0}(\mathbb{R}, X)
\end{aligned}
$$

for every $\omega(\cdot) \in C_{0}(\mathbb{R}, X)$.
According to (55), one has

$$
\begin{aligned}
& \left\|F_{2}(s, v(s)+\omega(s), B(v(s)+\omega(s)))\right\| \leq \beta(s) \\
& . \Phi\left(\|\omega(s)+B \omega(s)\|+\sup _{s \in \mathbb{R}}\|v(s)+B v(s)\|\right) \\
& \leq \beta(s) \Phi\left(\left(1+\|B\|_{\mathbb{L}(X)}\right)\|\omega(s)\|\right. \\
& \left.+\left(1+\|B\|_{\mathbb{L}(X)}\right) \sup _{s \in \mathbb{R}}\|v(s)\|\right) \leq \beta(s) \\
& . \Phi\left(\left(1+\|B\|_{\mathbb{L}(X)}\right)\left[\|\omega(s)\|+\sup _{s \in \mathbb{R}}\|v(s)\|\right]\right)
\end{aligned}
$$

for all $s \in \mathbb{R}$ and $\omega(s) \in X$ with $\|\omega(s)\| \leq r$; then

$$
\begin{aligned}
F_{2}(\cdot, v(\cdot)+\omega(\cdot), B(v(\cdot)+\omega(\cdot))) & \in C_{0}(\mathbb{R}, X) \\
& \text { as } \beta(\cdot) \in C_{0}\left(\mathbb{R}, \mathbb{R}^{+}\right) .
\end{aligned}
$$

Those, together with Lemma 21 , yield that $\Gamma$ is well defined and maps $C_{0}(\mathbb{R}, X)$ into itself.

On the other hand, in view of (55) and (98) it is not difficult to see that there exists a constant $k_{0}>0$ such that

$$
\begin{aligned}
& \frac{C M\|L\||\omega|^{-1 / \alpha} \pi\left(1+\|B\|_{\mathbb{Q}(X)}\right)}{\alpha \sin (\pi / \alpha)} k_{0} \\
& \quad+C M \rho_{2} \Phi\left(\left(1+\|B\|_{\mathbb{R}(X)}\right)\left(k_{0}+\sup _{s \in \mathbb{R}}\|v(s)\|\right)\right) \\
& \leq k_{0} .
\end{aligned}
$$

This enables us to conclude that, for any $t \in \mathbb{R}$ and $\omega_{1}(t)$, $\omega_{2}(t) \in \Omega_{k_{0}}$,

$$
\begin{aligned}
& \left\|\left(\Gamma^{1} \omega_{1}\right)(t)+\left(\Gamma^{2} \omega_{2}\right)(t)\right\| \leq \| \int_{-\infty}^{t} S_{\alpha}(t-s) \\
& \cdot\left[F_{1}\left(s, v(s)+\omega_{1}(s), B\left(v(s)+\omega_{1}(s)\right)\right)\right. \\
& \left.-F_{1}(s, v(s), B v(s))\right] \mathrm{d} s\|+\| \int_{-\infty}^{t} S_{\alpha}(t-s) \\
& \cdot F_{2}\left(s, v(s)+\omega_{2}(s), B\left(v(s)+\omega_{2}(s)\right)\right) \mathrm{d} s \| \\
& \leq \int_{-\infty}^{t} L(s)\left\|S_{\alpha}(t-s)\right\|\left[\left\|\omega_{1}(s)\right\|\right. \\
& \left.+\left\|B \omega_{1}(s)\right\|\right] \mathrm{d} s+C M \int_{-\infty}^{t} \frac{\beta(s)}{1+|\omega|(t-s)^{\alpha}} \Phi \\
& \cdot\left(\left\|\omega_{2}(s)\right\|+\left\|B \omega_{2}(s)\right\|+\|v(s)\|+\|B v(s)\|\right) \mathrm{d} s \\
& \leq C M \int_{-\infty}^{t} \frac{L(s)}{1+|\omega|(t-s)^{\alpha}}\left(1+\|B\|_{\mathbb{L}(X)}\right) \\
& \cdot\left\|\omega_{1}(s)\right\| \mathrm{d} s+C M \int_{-\infty}^{t} \frac{\beta(s)}{1+|\omega|(t-s)^{\alpha}} \Phi((1 \\
& \left.+\|B\|_{\mathbb{L}(X)}\right)\left(\left\|\omega_{2}(s)\right\|+\|v(s)\|\right) \mathrm{d} s \leq C M(1
\end{aligned}
$$




$$
\begin{aligned}
& \left.+\|B\|_{\mathbb{L}(X)}\right)\left\|\omega_{1}\right\|_{\infty} \int_{-\infty}^{t} \frac{L(s)}{1+|\omega|(t-s)^{\alpha}} \mathrm{d} s \\
& +C M \sigma(t) \Phi\left(( 1 + \| B \| _ { \mathbb { L } ( X ) } ) \left(\left\|\omega_{2}\right\|_{\infty}\right.\right. \\
& \left.\left.+\sup _{s \in \mathbb{R}}\|v(s)\|\right)\right) \\
& \leq C M\left(\sum_{m=0}^{+\infty} \int_{t-(m+1)}^{t-m} \frac{L(s)}{1+|\omega|(t-s)^{\alpha}} \mathrm{d} s\right)(1 \\
& \left.+\|B\|_{\mathbb{L}(X)}\right)\left\|\omega_{1}\right\|_{\infty}+C M \rho_{2} \Phi\left(\left(1+\|B\|_{\mathbb{L}(X)}\right)\right. \\
& \left.\cdot\left(\left\|\omega_{2}\right\|_{\infty}+\sup _{s \in \mathbb{R}}\|v(s)\|\right)\right) \\
& \leq C M\left(\sum_{m=0}^{+\infty} \frac{1}{1+|\omega| m^{\alpha}} \int_{t-(m+1)}^{t-m} L(s) \mathrm{d} s\right)(1 \\
& \left.+\|B\|_{\mathbb{L}(X)}\right)\left\|\omega_{1}\right\|_{\infty}+C M \rho_{2} \Phi\left(( 1 + \| B \| _ { \mathbb { L } ( X ) } ) \left(k_{0}\right.\right. \\
& \left.\left.+\sup _{s \in \mathbb{R}}\|v(s)\|\right)\right) \leq C M\left(\sum_{m=0}^{+\infty} \frac{1}{1+|\omega| m^{\alpha}}\right)\|L\|(1 \\
& \left.+\|B\|_{\mathbb{L}(X)}\right) k_{0}+C M \rho_{2} \Phi\left(( 1 + \| B \| _ { \mathbb { L } ( X ) } ) \left(k_{0}\right.\right.
\end{aligned}
$$

$$
\begin{aligned}
& \left.\left.+\sup _{s \in \mathbb{R}}\|v(s)\|\right)\right) \\
& =\frac{C M\|L\||\omega|^{-1 / \alpha} \pi\left(1+\|B\|_{\mathbb{L}(X)}\right)}{\alpha \sin (\pi / \alpha)} k_{0} \\
& +C M \rho_{2} \Phi\left(\left(1+\|B\|_{\mathbb{L}(X)}\right)\left(k_{0}+\sup _{s \in \mathbb{R}}\|v(s)\|\right)\right) \\
& \leq k_{0},
\end{aligned}
$$

which implies that $\left(\Gamma^{1} \omega_{1}\right)(t)+\left(\Gamma^{2} \omega_{2}\right)(t) \in \Omega_{k_{0}}$. Thus $\Gamma$ maps $\Omega_{k_{0}}$ into itself.

Step 3. Show that $\Gamma^{1}$ is a contraction on $\Omega_{k_{0}}$.

In fact, for any $\omega_{1}(t), \omega_{2}(t) \in \Omega_{k_{0}}$ and $t \in \mathbb{R}$, from (97) it follows that

$$
\begin{aligned}
\| & F_{1}\left(s, v(s)+\omega_{1}(s), B\left(v(s)+\omega_{1}(s)\right)\right) \\
& \left.-F_{1}(s, v(s), B v(s))\right] \\
& -\left[F_{1}\left(s, v(s)+\omega_{2}(s), B\left(v(s)+\omega_{2}(s)\right)\right)\right. \\
& \left.-F_{1}(s, v(s), B v(s))\right] \| \leq L(s)\left[\left\|\omega_{1}(s)-\omega_{2}(s)\right\|\right. \\
& \left.+\left\|B \omega_{1}(s)-B \omega_{2}(s)\right\|\right] \leq L(s)\left(1+\|B\|_{\mathbb{L}(X)}\right) \| \omega_{1}(s) \\
& -\omega_{2}(s) \| .
\end{aligned}
$$

Thus

$$
\begin{aligned}
& \left\|\left(\Gamma^{1} \omega_{1}\right)(t)-\left(\Gamma^{1} \omega_{2}\right)(t)\right\|=\| \int_{-\infty}^{t} S_{\alpha}(t-s)\left[\left(F_{1}\left(s, v(s)+\omega_{1}(s), B\left(v(s)+\omega_{1}(s)\right)\right)-F_{1}(s, v(s), B v(s))\right)\right. \\
& \left.-\left(F_{1}\left(s, v(s)+\omega_{2}(s), B\left(v(s)+\omega_{2}(s)\right)\right)-F_{1}(s, v(s), B v(s))\right)\right] \mathrm{d} s\left\|\leq \int_{-\infty}^{t} L(s)\right\| S_{\alpha}(t-s)\left\|\left(1+\|B\|_{\mathbb{L}(X)}\right)\right\| \omega_{1}(s) \\
& -\omega_{2}(s)\left\|\mathrm{d} s \leq C M \int_{-\infty}^{t} \frac{L(s)}{1+|\omega|(t-s)^{\alpha}}\left(1+\|B\|_{\mathbb{L}(X)}\right)\right\| \omega_{1}(s)-\omega_{2}(s) \| \mathrm{d} s \\
& \quad \leq C M\left(\sum_{m=0}^{+\infty} \int_{t-(m+1)}^{t-m} \frac{L(s)}{1+|\omega|(t-s)^{\alpha}} \mathrm{d} s\right)\left(1+\|B\|_{\mathbb{L}(X)}\right)\left\|\omega_{1}-\omega_{2}\right\|_{\infty} \leq C M\left(\sum_{m=0}^{+\infty} \frac{1}{1+|\omega| m^{\alpha}} \int_{t-(m+1)}^{t-m} L(s) \mathrm{d} s\right)(1 \\
& \left.+\|B\|_{\mathbb{L}(X)}\right)\left\|\omega_{1}-\omega_{2}\right\|_{\infty} \leq C M\left(\sum_{m=0}^{+\infty} \frac{1}{1+|\omega| m^{\alpha}}\right)\|L\|\left(1+\|B\|_{\mathbb{L}(X)}\right)\left\|\omega_{1}-\omega_{2}\right\|_{\infty} \\
& \quad=\frac{C M\|L\||\omega|^{-1 / \alpha} \pi\left(1+\|B\|_{\mathbb{L}(X)}\right)}{\alpha \sin (\pi / \alpha)}\left\|\omega_{1}-\omega_{2}\right\|_{\infty},
\end{aligned}
$$

which implies that

$$
\begin{aligned}
& \left\|\left(\Gamma^{1} \omega_{1}\right)(t)-\left(\Gamma^{1} \omega_{2}\right)(t)\right\|_{\infty} \\
& \quad \leq \frac{C M\|L\||\omega|^{-1 / \alpha} \pi\left(1+\|B\|_{\mathbb{L}(X)}\right)}{\alpha \sin (\pi / \alpha)}\left\|\omega_{1}-\omega_{2}\right\|_{\infty} .
\end{aligned}
$$

Thus, in view of (98), one obtains the conclusion.

Step 4. Show that $\Gamma^{2}$ is completely continuous on $\Omega_{k_{0}}$.

The proof is similar to the proof in Step 4 of Theorem 24.

Step 5. Show that (39) has at least one asymptotically almost automorphic mild solution. 
The proof is similar to the proof in Step 5 of Theorem 24.

Taking $A=-\rho^{\alpha} I$ with $\rho>0$ in (39), Theorem 27 gives the following corollary.

Corollary 28. Let $F: \mathbb{R} \times X \times X \longrightarrow X$ satisfy $\left(H_{1}^{\prime}\right)$ and $\left(H_{2}\right)$ with $L(t) \in B C\left(\mathbb{R}, \mathbb{R}^{+}\right)$. Put $\rho_{2}:=\sup _{t \in \mathbb{R}} \sigma(t)$. Let $\|L\|=$ $\sup _{t \in \mathbb{R}} \int_{t}^{t+1} L(s) \mathrm{d} s$. Then (39) admits at least one asymptotically almost automorphic mild solution whenever

$$
\frac{C\|L\| \rho \pi\left(1+\|B\|_{\mathbb{L}(X)}\right)}{\alpha \sin (\pi / \alpha)}+C \rho_{1} \rho_{2}\left(1+\|B\|_{\mathbb{L}(X)}\right)<1 .
$$

Now we consider a more general case of equations introducing a new class of functions $L(t)$. We have the following result.

$\left(H_{2}^{\prime}\right)$ There exists a function $\beta(t) \in C_{0}\left(\mathbb{R}, \mathbb{R}^{+}\right)$such that, for all $t \in \mathbb{R}$ and $x, y \in X$,

$$
\left\|F_{2}(t, x, y)\right\| \leq \beta(t)(\|x\|+\|y\|) .
$$

Theorem 29. Assume that $A$ is sectorial of type $\omega<0$. Let $F: \mathbb{R} \times X \times X \longrightarrow X$ satisfy the hypotheses $\left(H_{1}^{\prime}\right)$ and $\left(H_{2}^{\prime}\right)$ with $L(t) \in B C\left(\mathbb{R}, \mathbb{R}^{+}\right)$. Moreover the integral $\int_{-\infty}^{t} \max \{L(s), \beta(s)\} \mathrm{d} s$ exists for all $t \in \mathbb{R}$. Then (39) has at least one asymptotically almost automorphic mild solution.

Proof. The proof is divided into the following five steps.

Step 1. Define a mapping $\Lambda$ on $A A(\mathbb{R}, X)$ by (62) and prove that $\Lambda$ has a unique fixed point $v(t) \in A A(\mathbb{R}, X)$.

Firstly, similar to the proof in Step 1 of Theorem 27, we can prove that $\Lambda$ is well defined and maps $A P(\mathbb{R}, X)$ into itself; moreover $\Lambda$ is continuous.

Next, we prove that $\Lambda$ is a contraction on $A A(\mathbb{R}, X)$ and has a unique fixed point $v(t) \in A A(\mathbb{R}, X)$.

In fact, for $v_{1}(t), v_{2}(t)$ is in $A A(\mathbb{R}, X)$ and defines a new norm

$$
|\|x\||:=\sup _{t \in \mathbb{R}}\{\mu(t)\|x(t)\|\}
$$

where $\mu(t):=e^{-k \int_{-\infty}^{t} \max \{L(s), \beta(s)\} \mathrm{d} s}$ and $k$ is a fixed positive constant. Let $C_{\alpha}:=\sup _{t \in \mathbb{R}}\left\|S_{\alpha}(t)\right\|$; then we have

$$
\begin{aligned}
& \mu(t)\left\|\Lambda v_{1}(t)-\Lambda v_{2}(t)\right\|=\mu(t) \| \int_{-\infty}^{t} S_{\alpha}(t-\sigma) \\
& \cdot\left[F_{1}\left(\sigma, v_{1}(\sigma), B v_{1}(\sigma)\right)\right. \\
& \left.-F_{1}\left(\sigma, v_{2}(\sigma), B v_{2}(\sigma)\right)\right] \mathrm{d} \sigma \| \\
& \leq C_{\alpha} \int_{-\infty}^{t} \mu(t) L(\sigma)\left[\left\|v_{1}(\sigma)-v_{2}(\sigma)\right\|+\| B v_{1}(\sigma)\right. \\
& \left.-B v_{2}(\sigma) \|\right] \mathrm{d} \sigma=C_{\alpha} \int_{-\infty}^{t} \mu(t) \mu(\sigma) L(\sigma)
\end{aligned}
$$

$$
\begin{aligned}
& \cdot \mu(\sigma)^{-1}\left(1+\|B\|_{\mathbb{L}(X)}\right)\left\|v_{1}(\sigma)-v_{2}(\sigma)\right\| \mathrm{d} \sigma \\
& \leq C_{\alpha}\left(1+\|B\|_{\mathbb{L}(X)}\right)\left|\left\|v_{1}-v_{2} \mid\right\| \int_{-\infty}^{t} \mu(t) \mu(\sigma)^{-1}\right. \\
& \cdot L(\sigma) \mathrm{d} \sigma=\frac{C_{\alpha}\left(1+\|B\|_{\mathbb{L}(X)}\right)}{k}\left|\left\|v_{1}-v_{2} \mid\right\|\right. \\
& \cdot \int_{-\infty}^{t} k e^{-k \int_{\sigma}^{t} \max \{L(\tau), \beta(\tau)\} \mathrm{d} \tau} L(\sigma) \mathrm{d} \sigma \\
& \leq \frac{C_{\alpha}\left(1+\|B\|_{\mathbb{L}(X)}\right)}{k}\left|\left\|v_{1}-v_{2} \mid\right\|\right. \\
& \cdot \int_{-\infty}^{t} k e^{-k \int_{\sigma}^{t} L(\tau) \mathrm{d} \tau} L(\sigma) \mathrm{d} \sigma \\
& =\frac{C_{\alpha}\left(1+\|B\|_{\mathbb{L}(X)}\right)}{k}\left|\left\|v_{1}-v_{2} \mid\right\|\right. \\
& \cdot \int_{-\infty}^{t} \frac{\mathrm{d}}{\mathrm{d} \sigma}\left(e^{k \int_{t}^{\sigma} L(\tau) \mathrm{d} \tau}\right) \mathrm{d} \sigma \\
& =\frac{C_{\alpha}\left(1+\|B\|_{\mathbb{L}(X)}\right)}{k}\left(1-e^{-k \int_{-\infty}^{t} L(\tau) \mathrm{d} \tau}\right)\left|\left\|v_{1}-v_{2} \mid\right\|\right. \\
& \leq \frac{C_{\alpha}\left(1+\|B\|_{\mathbb{L}(X)}\right)}{k}\left|\left\|v_{1}-v_{2} \mid\right\|\right. \text {, }
\end{aligned}
$$

which implies that

$$
\|\| \Lambda x(t)-\Lambda y(t)\left|\left\|\leq \frac{C_{\alpha}\left(1+\|B\|_{\mathbb{L}(X)}\right)}{k}|\|x-y \mid\| .\right.\right.
$$

Hence $\Lambda$ has a unique fixed point $x \in A A(\mathbb{R}, X)$ when $k$ is greater than $C_{\alpha}\left(1+\|B\|_{\mathbb{L}(X)}\right)$.

Step 2. Set $\Theta_{r}:=\left\{\omega(t) \in C_{0}(\mathbb{R}, X):|\|\omega(t)\|| \leq r\right\}$. For the above $v(t)$, define $\Gamma:=\Gamma^{1}+\Gamma^{2}$ on $C_{0}(\mathbb{R}, X)$ as $(69)$ and prove that $\Gamma$ maps $\Theta_{k_{0}}$ into itself, where $k_{0}$ is a given constant.

Firstly, from (97) it follows that, for all $s \in \mathbb{R}, \omega(s) \in X$,

$$
\begin{aligned}
& \| F_{1}(s, v(s)+\omega(s), B(v(s)+\omega(s))) \\
& \quad-F_{1}(s, v(s), B v(s)) \| \leq L(s)[\|\omega(s)\|+\|B \omega(s)\|] \\
& \quad \leq L(s)\left(1+\|B\|_{\mathbb{L}(X)}\right)\|\omega(s)\|+\|B \omega(s)\|,
\end{aligned}
$$

which together with $L(s) \in B C\left(\mathbb{R}, \mathbb{R}^{+}\right)$implies that

$$
\begin{aligned}
& F_{1}(\cdot, v(\cdot)+\omega(\cdot), B(v(\cdot)+\omega(\cdot)))-F_{1}(\cdot, v(\cdot), B v(\cdot)) \\
& \quad \in C_{0}(\mathbb{R}, X) \text { for every } \omega(\cdot) \in C_{0}(\mathbb{R}, X)
\end{aligned}
$$

According to (114), one has

$$
\begin{aligned}
\left\|F_{2}(s, v(s)+\omega(s), B(v(s)+\omega(s)))\right\| \leq \beta(s) \\
\cdot(\|\omega(s)+B \omega(s)\|+\|v(s)+B v(s)\|) \leq \beta(s) \\
\cdot\left(\left(1+\|B\|_{\mathbb{L}(X)}\right)\|\omega(s)\|+\left(1+\|B\|_{\mathbb{L}(X)}\right)\|v(s)\|\right) \\
\quad \leq \beta(s)\left(\left(1+\|B\|_{\mathbb{L}(X)}\right)[\|\omega(s)\|+\|v(s)\|]\right)
\end{aligned}
$$


for all $s \in \mathbb{R}$ and $\omega(s) \in X$ with $\|\omega(s)\| \leq r$; then

$$
\begin{aligned}
F_{2}(\cdot, v(\cdot)+\omega(\cdot), B(v(\cdot)+\omega(\cdot))) & \in C_{0}(\mathbb{R}, X) \\
& \text { as } \beta(\cdot) \in C_{0}\left(\mathbb{R}, \mathbb{R}^{+}\right) .
\end{aligned}
$$

Those, together with Lemma 21 , yield that $\Gamma$ is well defined and maps $C_{0}(\mathbb{R}, X)$ into itself.

On the other hand, it is not difficult to see that there exists a constant $k_{0}>0$ such that

$$
\begin{aligned}
& \frac{2 C_{\alpha}\left(1+\|B\|_{\mathbb{L}(X)}\right)}{k} k_{0}+\frac{C_{\alpha}\left(1+\|B\|_{\mathbb{L}(X)}\right)}{k}|\|v(s) \mid\| \\
& \quad \leq k_{0}
\end{aligned}
$$

when $k$ is large enough. This enables us to conclude that, for any $t \in \mathbb{R}$ and $\omega_{1}(t), \omega_{2}(t) \in \Theta_{k_{0}}$,

$$
\begin{aligned}
& \mu(t)\left\|\left(\Gamma^{1} \omega_{1}\right)(t)+\left(\Gamma^{2} \omega_{2}\right)(t)\right\| \leq \mu(t) \| \int_{-\infty}^{t} S_{\alpha}(t-s) \\
& \cdot\left[F_{1}\left(s, v(s)+\omega_{1}(s), B\left(v(s)+\omega_{1}(s)\right)\right)\right. \\
& \left.-F_{1}(s, v(s), B v(s))\right] \mathrm{d} s\|+\mu(t)\| \int_{-\infty}^{t} S_{\alpha}(t \\
& -s) F_{2}\left(s, v(s)+\omega_{2}(s), B\left(v(s)+\omega_{2}(s)\right)\right) \mathrm{d} s \| \\
& \leq C_{\alpha} \int_{-\infty}^{t} \mu(t) L(s)\left(\left\|\omega_{1}(s)\right\|+\left\|B \omega_{1}(s)\right\|\right) \mathrm{d} s \\
& +C_{\alpha} \int_{-\infty}^{t} \mu(t) \beta(s)\left(\left\|\omega_{2}\right\|+\|v(s)\|+\left\|B \omega_{2}\right\|\right. \\
& +\|B v(s)\|) \mathrm{d} s=C_{\alpha} \int_{-\infty}^{t} \mu(t) \mu(s) L(s) \mu(s)^{-1} \\
& \cdot\left(1+\|B\|_{\mathbb{L}(X)}\right)\left\|\omega_{1}(s)\right\| \mathrm{d} s+C_{\alpha} \int_{-\infty}^{t} \mu(t) \mu(s) \\
& \cdot \beta(s) \mu(s)^{-1}\left(1+\|B\|_{\mathbb{L}(X)}\right)\left(\left\|\omega_{2}\right\|+\|v(s)\|\right) \mathrm{d} s \\
& \leq C_{\alpha}\left(1+\|B\|_{\mathbb{L}(X)}\right)\left|\left\|\omega_{1} \mid\right\| \int_{-\infty}^{t} \mu(t) \mu(s)^{-1} L(s) \mathrm{d} s\right. \\
& +C_{\alpha}\left(1+\|B\|_{\mathbb{L}(X)}\right)\left(\left|\left\|\omega _ { 2 } \left|\left\|+|\|v(s) \mid\|) \int_{-\infty}^{t} \mu(t)\right.\right.\right.\right.\right. \\
& \cdot \mu(s)^{-1} \beta(s) \mathrm{d} s=\frac{C_{\alpha}\left(1+\|B\|_{\mathbb{L}(X)}\right)}{k}\left|\left\|\omega_{1} \mid\right\|\right. \\
& \cdot \int_{-\infty}^{t} k e^{-k \int_{t}^{s} \max \{L(\tau), \beta(\tau)\} \mathrm{d} \tau} L(s) \mathrm{d} s+C_{\alpha}(1
\end{aligned}
$$

$$
\begin{gathered}
\mu(t)\left\|\left(\Gamma^{1} \omega_{1}\right)(t)-\left(\Gamma^{1} \omega_{2}\right)(t)\right\|=\mu(t) \| \int_{-\infty}^{t} S_{\alpha}(t-s)\left[\left(F_{1}\left(s, v(s)+\omega_{1}(s), B\left(v(s)+\omega_{1}(s)\right)\right)-F_{1}(s, v(s), B v(s))\right)\right. \\
\left.-\left(F_{1}\left(s, v(s)+\omega_{2}(s), B\left(v(s)+\omega_{2}(s)\right)\right)-F_{1}(s, v(s), B v(s))\right)\right] \mathrm{d} s\left\|\leq C_{\alpha} \int_{-\infty}^{t} \mu(t) L(\sigma)\left(1+\|B\|_{\mathbb{L}(X)}\right)\right\| \omega_{1}(\sigma)
\end{gathered}
$$

$$
\begin{aligned}
& \left.+\|B\|_{\mathbb{L}(X)}\right)\left(\left|\left\|\omega_{2}|\|+|\|v(s) \mid\|)\right.\right.\right. \\
& \cdot \int_{-\infty}^{t} k e^{-k \int_{t}^{s} \max \{L(\tau), \beta(\tau)\} \mathrm{d} \tau} \beta(s) \mathrm{d} s \\
& \leq \frac{C_{\alpha}\left(1+\|B\|_{\mathbb{L}(X)}\right)}{k}\left|\left\|\omega_{1} \mid\right\|\right. \\
& \cdot \int_{-\infty}^{t} k e^{-k \int_{t}^{s} L(\tau) \mathrm{d} \tau} L(s) \mathrm{d} s+C_{\alpha}\left(1+\|B\|_{\mathbb{L}(X)}\right) \\
& \cdot\left(||\left|\omega_{2}\right|||+|\|v(s) \mid\|) \int_{-\infty}^{t} k e^{-k \int_{t}^{s} \beta(\tau) \mathrm{d} \tau} \beta(s) \mathrm{d} s\right. \\
& =\frac{C_{\alpha}\left(1+\|B\|_{\mathbb{L}(X)}\right)}{k}\left|\left\|\omega_{1} \mid\right\| \int_{-\infty}^{t} \frac{\mathrm{d}}{\mathrm{d} s}\left(e^{k \int_{t}^{s} L(\tau) \mathrm{d} \tau}\right) \mathrm{d} s\right. \\
& +C_{\alpha}\left(1+\|B\|_{\mathbb{( X )}}\right)\left(\left|\left\|\omega_{2}|\|+|\|v(s) \mid\|)\right.\right.\right. \\
& \int_{-\infty}^{t} \frac{\mathrm{d}}{\mathrm{d} s}\left(e^{k \int_{t}^{s} \beta(\tau) \mathrm{d} \tau}\right) \mathrm{d} s \\
& =\frac{C_{\alpha}\left(1+\|B\|_{\mathbb{L}(X)}\right)}{k}\left(1-e^{-k \int_{-\infty}^{t} L(\tau) \mathrm{d} \tau}\right)\left|\left\|\omega_{1} \mid\right\|\right. \\
& +\frac{C_{\alpha}\left(1+\|B\|_{\mathbb{L}(X)}\right)}{k}\left(1-e^{-k \int_{-\infty}^{t} \beta(\tau) \mathrm{d} \tau}\right)\left(\left|\left\|\omega_{2} \mid\right\|\right.\right. \\
& +|\|v(s) \mid\|) \leq \frac{C_{\alpha}\left(1+\|B\|_{\mathbb{L}(X)}\right)}{k}\left|\left\|\omega_{1} \mid\right\|+\frac{C_{\alpha}}{k}(1\right. \\
& \left.+\|B\|_{\mathbb{L}(X)}\right)\left(\left|\left\|\omega _ { 2 } \left|\left\|+|\|v(s) \mid\|) \leq k_{0},\right.\right.\right.\right.\right.
\end{aligned}
$$

which implies that $\left(\Gamma^{1} \omega_{1}\right)(t)+\left(\Gamma^{2} \omega_{2}\right)(t) \in \Theta_{k_{0}}$. Thus $\Gamma$ maps $\Theta_{k_{0}}$ into itself.

Step 3. Show that $\Gamma^{1}$ is a contraction on $\Theta_{k_{0}}$.

In fact, for any $\omega_{1}(t), \omega_{2}(t) \in \Theta_{k_{0}}$ and $t \in \mathbb{R}$, from (97) it follows that

$$
\begin{aligned}
\| & F_{1}\left(s, v(s)+\omega_{1}(s), B\left(v(s)+\omega_{1}(s)\right)\right) \\
& \left.-F_{1}(s, v(s), B v(s))\right] \\
& -\left[F_{1}\left(s, v(s)+\omega_{2}(s), B\left(v(s)+\omega_{2}(s)\right)\right)\right. \\
& \left.-F_{1}(s, v(s), B v(s))\right] \| \leq L(s)\left[\left\|\omega_{1}(s)-\omega_{2}(s)\right\|\right. \\
& \left.+\left\|B \omega_{1}(s)-B \omega_{2}(s)\right\|\right] \leq L(s)\left(1+\|B\|_{\mathbb{L}(X)}\right) \\
& \cdot\left\|\omega_{1}(s)-\omega_{2}(s)\right\| .
\end{aligned}
$$

Thus 


$$
\begin{aligned}
& -\omega_{2}(\sigma)\left\|\mathrm{d} \sigma=C_{\alpha} \int_{-\infty}^{t} \mu(t) \mu(\sigma) L(\sigma) \mu(\sigma)^{-1}\left(1+\|B\|_{\mathbb{L}(X)}\right)\right\| \omega_{1}(\sigma)-\omega_{2}(\sigma)\left\|\mathrm{d} \sigma \leq C_{\alpha}\left(1+\|B\|_{\mathbb{L}(X)}\right) \mid\right\| \omega_{1} \\
& -\omega_{2}\left|\left\|\int_{-\infty}^{t} \mu(t) \mu(\sigma)^{-1} L(\sigma) \mathrm{d} \sigma=\frac{C_{\alpha}\left(1+\|B\|_{\mathbb{L}(X)}\right)}{k}\left|\left\|\omega_{1}-\omega_{2} \mid\right\| \int_{-\infty}^{t} k e^{-k \int_{\sigma}^{t} \max \{L(\tau), \beta(\tau)\} \mathrm{d} \tau} L(\sigma) \mathrm{d} \sigma\right.\right.\right. \\
& \leq \frac{C_{\alpha}\left(1+\|B\|_{\mathbb{L}(X)}\right)}{k}\left|\left\|\omega_{1}-\omega_{2}\left|\left\|\int_{-\infty}^{t} k e^{-k \int_{\sigma}^{t} L(\tau) \mathrm{d} \tau} L(\sigma) \mathrm{d} \sigma=\frac{C_{\alpha}\left(1+\|B\|_{\mathbb{L}(X)}\right)}{k}\left|\left\|\omega_{1}-\omega_{2} \mid\right\| \int_{-\infty}^{t} \frac{\mathrm{d}}{\mathrm{d} \sigma}\left(e^{k \int_{t}^{\sigma} L(\tau) \mathrm{d} \tau}\right) \mathrm{d} \sigma\right.\right.\right.\right.\right. \\
& =\frac{C_{\alpha}\left(1+\|B\|_{\mathbb{L}(X)}\right)}{k}\left(1-e^{-k \int_{-\infty}^{t} L(\tau) \mathrm{d} \tau}\right)\left|\left\|\omega_{1}-\omega_{2}\left|\left\|\leq \frac{C_{\alpha}\left(1+\|B\|_{\mathbb{L}(X)}\right)}{k}\left|\left\|\omega_{1}-\omega_{2} \mid\right\|,\right.\right.\right.\right.\right.
\end{aligned}
$$

which implies

$$
\begin{aligned}
& \|\|\left(\Gamma^{1} \omega_{1}\right)(t)-\left(\Gamma^{1} \omega_{2}\right)(t) \mid \| \\
& \quad \leq \frac{C_{\alpha}\left(1+\|B\|_{\mathbb{L}(X)}\right)}{k}\left|\left\|\omega_{1}-\omega_{2} \mid\right\| .\right.
\end{aligned}
$$

Thus, when $k$ is greater than $C_{\alpha}\left(1+\|B\|_{\mathbb{L}(X)}\right)$, one obtains the conclusion.

Step 4. Show that $\Gamma^{2}$ is completely continuous on $\Theta_{k_{0}}$.

Given $\varepsilon>0$. Let $\left\{\omega_{n}\right\}_{n=1}^{+\infty} \subset \Theta_{k_{0}}$ with $\omega_{n} \longrightarrow \omega_{0}$ in $\Theta_{k_{0}}$ as $n \longrightarrow+\infty$. Since $\sigma(t) \in C_{0}\left(\mathbb{R}, \mathbb{R}^{+}\right)$, one may choose a $t_{1}>0$ big enough such that, for all $t \geq t_{1}$,

$$
\left(1+\|B\|_{\mathbb{L}(X)}\right)\left(k_{0}+|\|v \mid\|) \sigma(t)<\frac{\varepsilon}{3 C M} .\right.
$$

Also, in view of $\left(H_{1}^{\prime}\right)$, we have

$$
\begin{aligned}
& F_{2}\left(s, v(s)+\omega_{k}(s), B\left(v(s)+\omega_{k}(s)\right)\right) \\
& \quad \longrightarrow F_{2}\left(s, v(s)+\omega_{0}(s), B\left(v(s)+\omega_{0}(s)\right)\right)
\end{aligned}
$$

for all $s \in\left(-\infty, t_{1}\right]$ as $k \longrightarrow+\infty$ and

$$
\begin{gathered}
\mu(\cdot) \| F_{2}\left(\cdot, v(\cdot)+\omega_{n}(\cdot), B\left(v(\cdot)+\omega_{n}(\cdot)\right)\right) \\
\left.-F_{2}\left(\cdot, v(\cdot)+\omega_{0}(\cdot), B v(\cdot)+\omega_{0}(\cdot)\right)\right) \| \leq \mu(\cdot) \\
\cdot \beta(\cdot)\left(\left\|\omega_{n}(\cdot)\right\|+\|v(\cdot)\|+\left\|B \omega_{n}(\cdot)\right\|+\|B v(\cdot)\|\right. \\
\left.+\left\|\omega_{0}(\cdot)\right\|+\|v(\cdot)\|+\left\|B \omega_{0}(\cdot)\right\|+\|B v(\cdot)\|\right) \leq \beta(\cdot) \\
\cdot\left(\left|\left\|\omega _ { n } \left|\left\|+\left|\left\|v \left|\left\|+\left|\left\|B \omega _ { n } \left|\left\|+\left|\left\|B v \left|\left\|+\left|\left\|\omega_{0} \mid\right\|\right.\right.\right.\right.\right.\right.\right.\right.\right.\right.\right.\right.\right.\right.\right.\right.\right.\right. \\
+\left|\left\|v \left|\left\|+\left|\left\|B \omega_{0}|\|+|\|B v \mid\|) \leq \beta(\cdot)\right.\right.\right.\right.\right.\right. \\
\cdot\left(2\left(1+\|B\|_{\mathbb{L}(X)}\right)\left(k_{0}+|\|v \mid\|)\right) \in L^{1}\left(-\infty, t_{1}\right] .\right.
\end{gathered}
$$

Hence, by the Lebesgue dominated convergence theorem we deduce that there exists an $N>0$ such that

$$
\begin{aligned}
C M & \int_{-\infty}^{t_{1}} \frac{1}{1+|\omega|(t-s)^{\alpha}} \mu(t) \\
\cdot & \| F_{2}\left(s, v(s)+\omega_{k}(s), B\left(v(s)+\omega_{k}(s)\right)\right)
\end{aligned}
$$

$$
\begin{aligned}
& -F_{2}\left(s, v(s)+\omega_{0}(s), B\left(v(s)+\omega_{0}(s)\right)\right) \| \mathrm{d} s \\
& \leq \frac{\varepsilon}{3}
\end{aligned}
$$

whenever $k \geq N$. Thus

$$
\begin{aligned}
& \mu(t)\left\|\left(\Gamma^{2} \omega_{k}\right)(t)-\left(\Gamma^{2} \omega_{0}\right)(t)\right\|=\mu(t) \| \int_{-\infty}^{t} S_{\alpha}(t-s) \\
& \cdot F_{2}\left(s, v(s)+\omega_{k}(s), B\left(v(s)+\omega_{k}(s)\right)\right) \mathrm{d} s \\
& -\int_{-\infty}^{t} \mathrm{~S}_{\alpha}(t-s) \\
& \cdot F_{2}\left(s, v(s)+\omega_{0}(s), B\left(v(s)+\omega_{0}(s)\right)\right) d s \| \\
& \leq C M \int_{-\infty}^{t_{1}} \frac{1}{1+|\omega|(t-s)^{\alpha}} \mu(t) \\
& \cdot \| F_{2}\left(s, v(s)+\omega_{k}(s), B\left(v(s)+\omega_{k}(s)\right)\right) \\
& -F_{2}\left(s, v(s)+\omega_{0}(s), B\left(v(s)+\omega_{0}(s)\right)\right) \| \mathrm{d} s \\
& +C M\left(2\left(1+\|B\|_{\mathbb{L}(X)}\right)\left(k_{0}+|\|v \mid\|)\right)\right. \\
& \cdot \int_{t_{1}}^{\max \left\{t, t_{1}\right\}} \frac{\beta(s)}{1+|\omega|(t-s)^{\alpha}} \mathrm{d} s \\
& \leq C M \int_{-\infty}^{t_{1}} \frac{1}{1+|\omega|(t-s)^{\alpha}} \mu(t) \\
& \cdot \| F_{2}\left(s, v(s)+\omega_{k}(s), B\left(v(s)+\omega_{k}(s)\right)\right) \\
& -F_{2}\left(s, v(s)+\omega_{0}(s), B\left(v(s)+\omega_{0}(s)\right)\right) \| \mathrm{d} s \\
& +C M \sigma(t)\left(2\left(1+\|B\|_{\mathbb{L}(X)}\right)\left(k_{0}+|\|v \mid\|)\right) \leq \frac{\varepsilon}{3}\right. \\
& +\frac{2 \varepsilon}{3}=\varepsilon
\end{aligned}
$$

whenever $k \geq N$. Accordingly, $\Gamma^{2}$ is continuous on $\Theta_{k_{0}}$. In the sequel, we consider the compactness of $\Gamma^{2}$. 
Set $B_{r}(X)$ for the closed ball with center at 0 and radius $r$ in $X, V=\Gamma^{2}\left(\Theta_{k_{0}}\right)$, and $z(t)=\Gamma^{2}(u(t))$ for $u(t) \in \Theta_{k_{0}}$. First, for all $\omega(t) \in \Theta_{k_{0}}$ and $t \in \mathbb{R}$,

$$
\begin{aligned}
& \mu(t)\left\|\left(\Gamma^{2} \omega\right)(t)\right\|=\mu(t) \| \int_{-\infty}^{t} S_{\alpha}(t-s) \\
& \cdot F_{2}(s, v(s)+\omega(s), B(v(s)+\omega(s))) \mathrm{d} s \| \\
& \leq C M \int_{-\infty}^{t} \frac{1}{1+|\omega|(t-s)^{\alpha}} \mu(t) \\
& \cdot\left\|F_{2}(s, v(s)+\omega(s), B(v(s)+\omega(s)))\right\| \mathrm{d} s \\
& \leq C M \int_{-\infty}^{t} \frac{\beta(s)}{1+|\omega|(t-s)^{\alpha}} \mu(t)(\|v(s)\|+\| \omega(s)) \\
& +\|B v(s)\|+\| B \omega(s)) \|) \mathrm{d} s \\
& \leq C M \int_{-\infty}^{t} \frac{\beta(s)}{1+|\omega|(t-s)^{\alpha}} \mu(t)\left(1+\|B\|_{\mathbb{L}(X)}\right)
\end{aligned}
$$

$$
\begin{aligned}
& \cdot(\|v(s)\|+\| \omega(s))) \mathrm{d} s \leq C M \sigma(t)(1 \\
& \left.+\|B\|_{\mathbb{L}(X)}\right)\left(k_{0}+|\|v(s) \mid\|)\right.
\end{aligned}
$$

in view of $\sigma(t) \in C_{0}\left(\mathbb{R}, \mathbb{R}^{+}\right)$which follows from Lemma 23; one concludes that

$$
\lim _{|t| \longrightarrow+\infty}\left(\Gamma^{2} \omega\right)(t)=0 \quad \text { uniformly for } \omega(t) \in \Theta_{k_{0}} .
$$

as

$$
\begin{aligned}
& \left(\Gamma^{2} \omega\right)(t)=\int_{-\infty}^{t} S_{\alpha}(t-s) F_{2}(s, v(s) \\
& \quad+\omega(s), B(v(s)+\omega(s))) \mathrm{d} s=\int_{0}^{+\infty} S_{\alpha}(\tau) \\
& \quad \cdot F_{2}(t-\tau, v(t-\tau) \\
& \quad+\omega(t-\tau), B(v(t-\tau)+\omega(t-\tau))) \mathrm{d} \tau .
\end{aligned}
$$

Hence, for given $\varepsilon_{0}>0$, one can choose a $\xi>0$ such that

$$
\|\left|\int_{\xi}^{+\infty} S_{\alpha}(\tau) F_{2}(t-\tau, v(t-\tau)+\omega(t-\tau), B(v(t-\tau)+\omega(t-\tau))) \mathrm{d} \tau\right|||<\varepsilon_{0}
$$

Thus we get

$$
z(t) \in \overline{\xi c\left(\left\{S_{\alpha}(\tau) F_{2}(\lambda, v(\lambda)+\omega(\lambda), B(v(\lambda)+\omega(\lambda))): 0 \leq \tau \leq \xi, t-\xi \leq \lambda \leq \xi, \quad\left|\|\omega \mid\| \leq k_{0}\right\}\right)\right.}+B_{\varepsilon_{0}}\left(\Theta_{k_{0}}\right),
$$

where $c(K)$ denotes the convex hull of $K$. Using the fact that $S_{\alpha}(\cdot)$ is strongly continuous, we infer that

$$
\begin{aligned}
K & =\left\{S_{\alpha}(\tau) F_{2}(\lambda, v(\lambda)+\omega(\lambda), B(v(\lambda)+\omega(\lambda))): 0\right. \\
& \leq \tau \leq \xi, t-\xi \leq \lambda \leq \xi, \quad\left|\|\omega \mid\| \leq k_{0}\right\}
\end{aligned}
$$

Next, we verify the equicontinuity of the set $\left\{\left(\Gamma^{2} \omega\right)(t)\right.$ : $\left.\omega(t) \in \Theta_{k_{0}}\right\}$, given $\varepsilon_{1}>0$. In view of (114), together with the continuity of $\left\{S_{\alpha}(t)\right\}_{t>0}$, there exists an $\eta>0$ such that, for all $\omega(t) \in \Omega_{k_{0}}$ and $t_{2} \geq t_{1}$ with $t_{2}-t_{1}<\eta$,

is a relatively compact set and $V \subset \overline{\xi(K)}+B_{\varepsilon_{0}}\left(\Theta_{k_{0}}\right)$, which implies that $V$ is a relatively compact subset of $\Theta_{k_{0}}$.

$$
\begin{array}{r}
\int_{t_{1}}^{t_{2}}\left|\left\|S_{\alpha}\left(t_{2}-s\right) F_{2}(s, v(s)+\omega(s), B(v(s)+\omega(s))) \mid\right\| \mathrm{d} s<\frac{\varepsilon_{1}}{4},\right. \\
\int_{t_{1}-\eta}^{t_{1}}\left|\left\|\left[S_{\alpha}\left(t_{2}-s\right)-S_{\alpha}\left(t_{1}-s\right)\right] F_{2}(s, v(s)+\omega(s), B(v(s)+\omega(s))) \mid\right\| \mathrm{d} s<\frac{\varepsilon_{1}}{4} .\right.
\end{array}
$$

Also, one can choose a $k>0$ such that

$$
\begin{gathered}
\int_{t_{1}-k}^{t_{1}-\eta}\left|\left\|\left[S_{\alpha}\left(t_{2}-s\right)-S_{\alpha}\left(t_{1}-s\right)\right] F_{2}(s, v(s)+\omega(s), B(v(s)+\omega(s))) \mid\right\| \mathrm{d} s<\frac{\varepsilon_{1}}{4}\right. \\
\left(1+\|B\|_{\mathbb{L}(X)}\right)\left(k_{0}+|\|v \mid\|) \sup _{s \in\left[-\infty, t_{1}-k\right]}\left\|S_{\alpha}\left(t_{2}-s\right)-S_{\alpha}\left(t_{1}-s\right)\right\| \int_{-\infty}^{t_{1}-k} \beta(s) \mathrm{d} s<\frac{\varepsilon_{1}}{4},\right.
\end{gathered}
$$


which implies that, for all $\omega(t) \in \Omega_{k_{0}}$ and $t_{2} \geq t_{1}$,

$$
\begin{aligned}
& \int_{-\infty}^{t_{1}-k}\left|\left\|\left[S_{\alpha}\left(t_{2}-s\right)-S_{\alpha}\left(t_{1}-s\right)\right] F_{2}(s, v(s)+\omega(s), B(v(s)+\omega(s))) \mid\right\| \mathrm{d} s\right. \\
& \quad \leq\left(1+\|B\|_{\mathbb{L}(X)}\right)\left(k_{0}+|\|v \mid\|) \sup _{s \in\left[-\infty, t_{1}-k\right]}\left\|S_{\alpha}\left(t_{2}-s\right)-S_{\alpha}\left(t_{1}-s\right)\right\| \int_{-\infty}^{t_{1}-k} \beta(s) \mathrm{d} s<\frac{\varepsilon_{1}}{4} .\right.
\end{aligned}
$$

Then one has

$$
\begin{aligned}
& \|\|\left(\Gamma^{2} \omega\right)\left(t_{2}\right)-\left(\Gamma^{2} \omega\right)\left(t_{1}\right) \mid \| \\
& \quad=\left|\left\|\int_{-\infty}^{t_{2}} S_{\alpha}\left(t_{2}-s\right) F_{2}(s, v(s)+\omega(s), B(v(s)+\omega(s))) \mathrm{d} s-\int_{-\infty}^{t_{1}} S_{\alpha}\left(t_{1}-s\right) F_{2}(s, v(s)+\omega(s), B(v(s)+\omega(s))) \mathrm{d} s \mid\right\|\right. \\
& \quad \leq \int_{t_{1}}^{t_{2}}\left|\left\|S_{\alpha}\left(t_{2}-s\right) F_{2}(s, v(s)+\omega(s), B(v(s)+\omega(s))) \mid\right\| \mathrm{d} s\right. \\
& \quad+\int_{t_{1}-\eta}^{t_{1}}\left|\left\|\left[S_{\alpha}\left(t_{2}-s\right)-S_{\alpha}\left(t_{1}-s\right)\right] F_{2}(s, v(s)+\omega(s), B(v(s)+\omega(s))) \mid\right\| \mathrm{d} s\right. \\
& \quad+\int_{-\infty}^{t_{1}-k}||\left|\left[S_{\alpha}\left(t_{2}-s\right)-S_{\alpha}\left(t_{1}-s\right)\right] F_{2}(s, v(s)+\omega(s), B(v(s)+\omega(s)))\right| \| \mathrm{d} s \\
& \quad+\int_{t_{1}-k}^{t_{1}-\eta}\left|\left\|\left[S_{\alpha}\left(t_{2}-s\right)-S_{\alpha}\left(t_{1}-s\right)\right] F_{2}(s, v(s)+\omega(s), B(v(s)+\omega(s))) \mid\right\| \mathrm{d} s<\varepsilon_{1}\right.
\end{aligned}
$$

which implies the equicontinuity of the set $\left\{\left(\Gamma^{2} \omega\right)(t): \omega(t) \epsilon\right.$ $\left.\Theta_{k_{0}}\right\}$.

Now an application of Lemma 18 justifies the compactness of $\Gamma^{2}$.

Step 5. Show that (39) has at least one asymptotically almost automorphic mild solution.

The proof is similar to the proof in Step 5 of Theorem 24.

Taking $A=-\rho^{\alpha} I$ with $\rho>0$ in (39), Theorem 29 gives the following corollary.

Corollary 30. Let $F: \mathbb{R} \times X \times X \longrightarrow X$ satisfy $\left(H_{1}^{\prime}\right)$ and $\left(H_{2}^{\prime}\right)$ with $L(t) \in B C\left(\mathbb{R}, \mathbb{R}^{+}\right)$. Moreover the integral $\int_{-\infty}^{t} \max \{L(s), \beta(s)\} \mathrm{d} s$ exists for all $t \in \mathbb{R}$. Then (39) has at least one asymptotically almost automorphic mild solution.

\section{Applications}

In this section we give an example to illustrate the above results.

Consider the following fractional relaxation-oscillation equation:

$$
\begin{aligned}
\partial_{t}^{\alpha} u(t, x) & =\partial_{x}^{2} u(t, x)-p u(t, x) \\
+\partial_{t}^{\alpha-1} & {\left[\mu a(t) \sin \left(\frac{1}{2+\cos t+\cos \sqrt{2} t}\right)\right.}
\end{aligned}
$$

$$
\begin{aligned}
& \times[\sin u(t, x)+u(t, x)] \\
& \left.+v e^{-|t|}[u(t, x)+\sin u(t, x)]\right],
\end{aligned}
$$$$
t \in \mathbb{R}, x \in[0, \pi],
$$$$
u(t, 0)=u(t, \pi)=0, \quad t \in \mathbb{R},
$$

where $a(t) \in B C\left(\mathbb{R}, \mathbb{R}^{+}\right)$is a function and $p, \mu$, and $\nu$ are positive constants.

Take $X=L^{2}([0, \pi])$ and define the operator $A$ by

$$
A \varphi:=\varphi^{\prime \prime}-p \varphi, \quad \varphi \in D(A),
$$

where

$$
D(A):=\left\{\varphi \in X: \varphi^{\prime \prime} \in X, \varphi(0)=\varphi(\pi)\right\} \subset X .
$$

It is well known that $B u=u^{\prime \prime}$ is self-adjoint, with compact resolvent, and is the infinitesimal generator of an analytic semigroup on $X$. Hence, $p I-B$ is sectorial of type $\omega=-p<0$. Let

$$
\begin{aligned}
& F_{1}(t, x(\xi), y(\xi)):=\mu a(t) \\
& \cdot \sin \left(\frac{1}{2+\cos t+\cos \sqrt{2} t}\right)[\sin x(\xi)+y(\xi)], \\
& F_{2}(t, x(\xi), y(\xi)):=v e^{-|t|}[x(\xi)+\sin y(\xi)] .
\end{aligned}
$$


Then it is easy to verify that $F_{1}, F_{2}: \mathbb{R} \times X \times X \rightarrow X$ are continuous and $F_{1}(t, x, y) \in A A(\mathbb{R} \times X \times X, X)$ satisfying

$$
\begin{aligned}
& \left\|F_{1}\left(t, x_{1}, y_{1}\right)-F_{1}\left(t, x_{2}, y_{2}\right)\right\|_{2}^{2} \\
& \leq \int_{0}^{\pi} \mu^{2}\left|a(t) \sin \left(\frac{1}{2+\cos t+\cos \sqrt{2} t}\right)\right|^{2} \\
& \cdot\left|\left[\sin x_{1}(s)+y_{1}(s)\right]-\left[\sin x_{2}(s)+y_{2}(s)\right]\right| \mathrm{d} s \\
& \leq \mu^{2} a^{2}(t)\left|\sin \left(\frac{1}{2+\cos t+\cos \sqrt{2} t}\right)\right|^{2} \\
& \cdot\left(\left\|x_{1}-x_{2}\right\|_{2}^{2}+\left\|y_{1}-y_{2}\right\|_{2}^{2}\right),
\end{aligned}
$$

that is,

$$
\begin{aligned}
& \left\|F_{1}\left(t, x_{1}, y_{1}\right)-F_{1}\left(t, x_{2}, y_{2}\right)\right\|_{2} \\
& \leq \mu a(t)\left(\left\|x_{1}-x_{2}\right\|_{2}+\left\|y_{1}-y_{2}\right\|_{2}\right) \\
& \quad \forall t \in \mathbb{R}, x_{1}, y_{1}, x_{2}, y_{2} \in X ;
\end{aligned}
$$

furthermore

$$
\begin{aligned}
& \left\|F_{1}\left(t, x_{1}, y_{1}\right)-F_{1}\left(t, x_{2}, y_{2}\right)\right\|_{2} \\
& \leq \mu\|a\|_{\infty}\left(\left\|x_{1}-x_{2}\right\|_{2}+\left\|y_{1}-y_{2}\right\|_{2}\right) \\
& \forall t \in \mathbb{R}, x_{1}, y_{1}, x_{2}, y_{2} \in X .
\end{aligned}
$$

And

$$
\begin{aligned}
\left\|F_{2}(t, x, y)\right\|_{2}^{2} & \leq \int_{0}^{\pi} v^{2} e^{-2|t|}|x(s)+\sin y(s)| \mathrm{d} s \\
& \leq v^{2} e^{-2|t|}\left(\|x\|_{2}^{2}+\|y\|_{2}^{2}\right),
\end{aligned}
$$

that is,

$$
\begin{aligned}
&\left\|F_{2}(t, x, y)\right\|_{2} \leq v e^{-|t|}\left(\|x\|_{2}+\|y\|_{2}\right) \\
& \forall t \in \mathbb{R}, x, y \in X,
\end{aligned}
$$

which implies $F_{2}(t, x, y) \in C_{0}(\mathbb{R} \times X \times X, X)$. Furthermore

$$
\begin{aligned}
F(t, x, y) & =F_{1}(t, x, y)+F_{2}(t, x, y) \\
& \in A A A(\mathbb{R} \times X \times X, X) .
\end{aligned}
$$

Thus, (142) can be reformulated as the abstract problem (39) and the assumptions $\left(H_{1}\right)$ and $\left(H_{2}\right)$ hold with

$$
\begin{aligned}
L & =\mu\|a\|_{\infty}, \\
\Phi(r) & =r, \\
\beta(t) & =v e^{-|t|}, \\
\rho_{1} & =1, \\
\rho_{2} & \leq v,
\end{aligned}
$$

the assumption $\left(H_{1}^{\prime}\right)$ holds with $L(t)=\mu a(t)$, and the assumption $\left(H_{2}^{\prime}\right)$ holds.
In consequence, the fractional relaxation-oscillation equation (142) has at least one asymptotically almost automorphic mild solutions if either

$$
\frac{\mu C M\|a\|_{\infty} \pi|p|^{-1 / \alpha}}{\alpha \sin (\pi / \alpha)}+C M \nu<\frac{1}{2}
$$

(Theorem 24) or

$$
\frac{\mu C M\|a\| \pi|p|^{-1 / \alpha}}{\alpha \sin (\pi / \alpha)}+C M \nu<\frac{1}{2}
$$

(Theorem 27), where $\|a\|=\sup _{t \in \mathbb{R}} \int_{t}^{t+1} a(s) \mathrm{d} s$ or the integral

$$
\int_{-\infty}^{t} \max \left\{\mu a(s), v e^{-|t|}\right\} \mathrm{d} s
$$

exists for all $t \in \mathbb{R}$ (Theorem 29).

\section{Conflicts of Interest}

The authors declare that they have no conflicts of interest.

\section{Acknowledgments}

This research was supported by the NNSF of China (no. 11561009) and (no. 41665006), the Guangdong Province Natural Science Foundation (no. 2015A030313896), the Characteristic Innovation Project (Natural Science) of Guangdong Province (no. 2016KTSCX094), the Science and Technology Program Project of Guangzhou (no. 201707010230), and the Guangxi Province Natural Science Foundation (no. 2016GXNSFAA380240).

\section{References}

[1] S. Bochner, "Continuous mappings of almost automorphic and almost periodic functions," Proceedings of the National Acadamy of Sciences of the United States of America, vol. 52, pp. 907-910, 1964.

[2] S. Bochner, "Uniform convergence of monotone sequences of functions," Proceedings of the National Acadamy of Sciences of the United States of America, vol. 47, pp. 582-585, 1961.

[3] S. Bochner, "A new approach to almost periodicity," Proceedings of the National Acadamy of Sciences of the United States of America, vol. 48, pp. 2039-2043, 1962.

[4] S. Bochner and J. Von Neumann, "On compact solutions of operational-differential equations," I. Annals of Mathematics: Second Series, vol. 36, no. 1, pp. 255-291, 1935.

[5] G. M. N'Guérékata, Almost Automorphic Functions and Almost Periodic Functions in Abstract Spaces, Kluwer Academic/Plenum Publishers, New York, London, Moscow, 2001.

[6] G. M. N'Guerekata, Topics in Almost Automorphy, Springer, New York, NY, USA, 2005.

[7] W. A. Veech, "Almost automorphic functions," Proceedings of the National Acadamy of Sciences of the United States of America, vol. 49, pp. 462-464, 1963.

[8] J. Campos and M. Tarallo, "Almost automorphic linear dynamics by Favard theory," Journal of Differential Equations, vol. 256, no. 4, pp. 1350-1367, 2014. 
[9] T. Caraballo and D. Cheban, "Almost periodic and almost automorphic solutions of linear differential/difference equations without Favard's separation condition," I. Journal of Differential Equations, vol. 246, no. 1, pp. 108-128, 2009.

[10] L. Mahto and S. Abbas, "PC-almost automorphic solution of impulsive fractional differential equations," Mediterranean Journal of Mathematics, vol. 12, no. 3, pp. 771-790, 2015.

[11] D. Araya and C. Lizama, "Almost automorphic mild solutions to fractional differential equations," Nonlinear Analysis. Theory, Methods \& Applications. An International Multidisciplinary Journal, vol. 69, no. 11, pp. 3692-3705, 2008.

[12] G. M. Mophou and G. M. N’Guérékata, “On some classes of almost automorphic functions and applications to fractional differential equations," Computers \& Mathematics with Applications. An International Journal, vol. 59, no. 3, pp. 1310-1317, 2010.

[13] L. Abadias and C. Lizama, "Almost automorphic mild solutions to fractional partial difference-differential equations," Applicable Analysis: An International Journal, vol. 95, no. 6, pp. 13471369, 2016.

[14] M. Fu and Z. Liu, "Square-mean almost automorphic solutions for some stochastic differential equations," Proceedings of the American Mathematical Society, vol. 138, no. 10, pp. 3689-3701, 2010.

[15] J. Cao, Q. Yang, and Z. Huang, "Existence and exponential stability of almost automorphic mild solutions for stochastic functional differential equations," Stochastics. An International Journal of Probability and Stochastic Processes, vol. 83, no. 3, pp. 259-275, 2011.

[16] Z. Liu and K. Sun, "Almost automorphic solutions for stochastic differential equations driven by Lévy noise," Journal of Functional Analysis, vol. 266, no. 3, pp. 1115-1149, 2014.

[17] G. M. N'guérékata, "Comments on almost automorphic and almost periodic functions in Banach spaces," Far East Journal of Mathematical Sciences (FJMS), vol. 17, no. 3, pp. 337-344, 2005.

[18] G. M. N'Guérékata, "Sur les solutions presqu automorphes d'équations différentielles abstraites," Annales des Sciences Mathématiques du Québec, no. 1, pp. 69-79, 1981.

[19] D. Bugajewski and G. M. N'Guérékata, “On the topological structure of almost automorphic and asymptotically almost automorphic solutions of differential and integral equations in abstract spaces," Nonlinear Analysis: Theory, Methods \& Applications, vol. 59, no. 8, pp. 1333-1345, 2004.

[20] T. Diagana, E. M. Hernández, and J. dos Santos, "Existence of asymptotically almost automorphic solutions to some abstract partial neutral integro-differential equations," Nonlinear Analysis. Theory, Methods \& Applications. An International Multidisciplinary Journal, vol. 71, no. 1-2, pp. 248-257, 2009.

[21] H.-S. Ding, T.-J. Xiao, and J. Liang, "Asymptotically almost automorphic solutions for some integrodifferential equations with nonlocal initial conditions," Journal of Mathematical Analysis and Applications, vol. 338, no. 1, pp. 141-151, 2008.

[22] J.-Q. Zhao, Y.-K. Chang, and G. M. N'guérékata, "Existence of asymptotically almost automorphic solutions to nonlinear delay integral equations," Dynamic Systems and Applications, vol. 21, no. 2-3, pp. 339-349, 2012.

[23] Y.-K. Chang and C. Tang, "Asymptotically almost automorphic solutions to stochastic differential equations driven by a Lévy process," Stochastics. An International Journal of Probability and Stochastic Processes, vol. 88, no. 7, pp. 980-1011, 2016.

[24] Z.-H. Zhao, Y.-K. Chang, and J. J. Nieto, "Square-mean asymptotically almost automorphic process and its application to stochastic integro-differential equations," Dynamic Systems and Applications, vol. 22, no. 2-3, pp. 269-284, 2013.

[25] G. M. N'Guérékata, Spectral Theory for Bounded Functions and Applications to Evolution Equations, Nova Science Publishers, NY, USA, 2017.

[26] A. A. Kilbas, H. M. Srivastava, and J. J. Trujillo, Theory and Applications of Fractional Differential Equations, vol. 24 of North-Holland Mathematics Studies, Elsevier Science B.V., Amsterdam, 2006.

[27] S. G. Samko, A. A. Kilbas, and O. I. Marichev, Fractional integral and derivatives: Theory and applications, Gordon and Breach Science Publishers, Switzerland, 1993.

[28] K. Diethelm, The Analysis of Fractional Differential Equations, Lecture Notes in Mathematics, Springer Verlag, Berlin, Heidelberg, Germany, 2010.

[29] R. Hilfer, Applications of Fractional Calculus in Physics, World Scientific, Singapore, 2000.

[30] I. Podlubny, Fractional Differential Equations, Academic Press, CA, USA, 1999.

[31] K. S. Miller and B. Ross, An Introduction to the Fractional Calculus and Fractional Differential Equations, A Wiley Interscience Publication, John Wiley \& Sons, NY, USA, 1993.

[32] Y. Zhou, Basicheory of Fractional Diferential Equations, World Scientiic, Singapore, 2014.

[33] R. P. Agarwal, M. Belmekki, and M. Benchohra, "A survey on semilinear differential equations and inclusions involving Riemann-Liouville fractional derivative," Advances in Difference Equations, vol. 2009, Article ID 981728, 47 pages, 2009.

[34] R. P. Agarwal, V. Lakshmikantham, and J. J. Nieto, "On the concept of solution for fractional differential equations with uncertainty," Nonlinear Analysis. Theory, Methods \& Applications. An International Multidisciplinary Journal, vol. 72, no. 6, pp. 2859-2862, 2010.

[35] M. Benchohra, J. Henderson, S. K. Ntouyas, and A. Ouahab, "Existence results for fractional order functional differential equations with infinite delay," Journal of Mathematical Analysis and Applications, vol. 338, no. 2, pp. 1340-1350, 2008.

[36] R. P. Agarwal, M. Benchohra, and S. Hamani, "A survey on existence results for boundary value problems of nonlinear fractional differential equations and inclusions," Acta Applicandae Mathematicae, vol. 109, no. 3, pp. 973-1033, 2010.

[37] M. M. El-Borai, "Some probability densities and fundamental solutions of fractional evolution equations," Chaos, Solitons \& Fractals, vol. 14, no. 3, pp. 433-440, 2002.

[38] V. Lakshmikantham, "Theory of fractional functional differential equations," Nonlinear Analysis: Theory, Methods \& Applications, vol. 60, pp. 3337-3343, 2008.

[39] V. Lakshmikantham and A. S. Vatsala, "Basic theory of fractional differential equations," Nonlinear Analysis. Theory, Methods \& Applications. An International Multidisciplinary Journal, vol. 69, no. 8, pp. 2677-2682, 2008.

[40] V. Lakshmikantham and A. S. Vatsala, "Theory of fractional differential inequalities and applications," Communications in Applied Analysis, vol. 11, no. 3-4, pp. 395-402, 2007.

[41] V. Lakshmikantham and J. V. Devi, "Theory of fractional differential equations in a Banach space," European Journal of Pure and Applied Mathematics, vol. 1, no. 1, pp. 38-45, 2008.

[42] G. Mophou, O. Nakoulima, and G. M. N’Guérékata, “Existence results for some fractional differential equations with nonlocal conditions," Nonlinear Studies. The International Journal, vol. 17, no. 1, pp. 15-21, 2010. 
[43] G. M. Mophou and G. M. N’Guérékata, “Existence of the mild solution for some fractional differential equations with nonlocal conditions," Semigroup Forum, vol. 79, no. 2, pp. 315-322, 2009.

[44] G. M. Mophou and G. M. N’Guérékata, “On integral solutions of some nonlocal fractional differential equations with nondense domain," Nonlinear Analysis. Theory, Methods \& Applications. An International Multidisciplinary Journal, vol. 71, no. 10, pp. 4668-4675, 2009.

[45] G. M. Mophou, "Existence and uniqueness of mild solutions to impulsive fractional differential equations," Nonlinear Analysis. Theory, Methods \& Applications. An International Multidisciplinary Journal, vol. 72, no. 3-4, pp. 1604-1615, 2010.

[46] G. M. N’Guérékata, “A Cauchy problem for some fractional abstract differential equation with non local conditions," Nonlinear Analysis. Theory, Methods \& Applications. An International Multidisciplinary Journal, vol. 70, no. 5, pp. 1873-1876, 2009.

[47] Y. Zhou and L. Peng, "On the time-fractional Navier-Stokes equations," Computers \& Mathematics with Applications. An International Journal, vol. 73, no. 6, pp. 874-891, 2017.

[48] Y. Zhou and L. Peng, "Weak solutions of the time-fractional Navier-Stokes equations and optimal control," Computers \& Mathematics with Applications. An International Journal, vol. 73, no. 6, pp. 1016-1027, 2017.

[49] Y. Zhou and L. Zhang, "Existence and multiplicity results of homoclinic solutions for fractional Hamiltonian systems," Computers \& Mathematics with Applications. An International Journal, vol. 73, no. 6, pp. 1325-1345, 2017.

[50] Y. Zhou, V. Vijayakumar, and R. Murugesu, "Controllability for fractional evolution inclusions without compactness," Evolution Equations and Control Theory, vol. 4, no. 4, pp. 507-524, 2015.

[51] C. Cuevas and C. Lizama, "Almost automorphic solutions to a class of semilinear fractional differential equations," Applied Mathematics Letters, vol. 21, no. 12, pp. 1315-1319, 2008.

[52] R. P. Agarwal, B. de Andrade, and C. Cuevas, "On type of periodicity and ergodicity to a class of fractional order differential equations," Advances in Difference Equations, Article ID 179750, 2010.

[53] R. P. Agarwal, C. Cuevas, and H. Soto, "Pseudo-almost periodic solutions of a class of semilinear fractional differential equations," Applied Mathematics and Computation, vol. 37, no. 1-2, pp. 625-634, 2011.

[54] R. P. Agarwal, B. de Andrade, and C. Cuevas, "Weighted pseudo-almost periodic solutions of a class of semilinear fractional differential equations," Nonlinear Analysis: Real World Applications, vol. 11, no. 5, pp. 3532-3554, 2010.

[55] H.-S. Ding, J. Liang, and T.-J. Xiao, "Almost automorphic solutions to abstract fractional differential equations," Advances in Difference Equations, Article ID 508374, 2010.

[56] C. Cuevas, A. Sepúlveda, and H. Soto, "Almost periodic and pseudo-almost periodic solutions to fractional differential and integro-differential equations," Applied Mathematics and Computation, vol. 218, no. 5, pp. 1735-1745, 2011.

[57] Y.-K. Chang, R. Zhang, and G. M. N’Guérékata, "Weighted pseudo almost automorphic mild solutions to semilinear fractional differential equations," Computers \& Mathematics with Applications. An International Journal, vol. 64, no. 10, pp. 31603170, 2012.

[58] B. He, J. Cao, and B. Yang, "Weighted Stepanov-like pseudoalmost automorphic mild solutions for semilinear fractional differential equations," Advances in Difference Equations, vol. 2015, 74 pages, 2015.
[59] J. Cao, Q. Yang, and Z. Huang, "Existence of anti-periodic mild solutions for a class of semilinear fractional differential equations," Communications in Nonlinear Science and Numerical Simulation, vol. 17, no. 1, pp. 277-283, 2012.

[60] C. Lizama and F. Poblete, "Regularity of mild solutions for a class of fractional order differential equations," Applied Mathematics and Computation, vol. 224, pp. 803-816, 2013.

[61] Z. Xia, M. Fan, and R. P. Agarwal, "Pseudo almost automorphy of semilinear fractional differential equations in Banach spaces," Fractional Calculus and Applied Analysis, vol. 19, no. 3, pp. 741764, 2016.

[62] S. Abbas, V. Kavitha, and R. Murugesu, "Stepanov-like weighted pseudo almost automorphic solutions to fractional order abstract integro-differential equations," Journal of Fractional Calculus and Applications, vol. 4, pp. 1-19, 2013.

[63] G. M. Mophou, "Weighted pseudo almost automorphic mild solutions to semilinear fractional differential equations," Applied Mathematics and Computation, vol. 217, no. 19, pp. 7579-7587, 2011.

[64] Y.-K. Chang and X.-X. Luo, "Pseudo almost automorphic behavior of solutions to a semi-linear fractional differential equation," Mathematical Communications, vol. 20, no. 1, pp. 5368, 2015.

[65] V. Kavitha, S. Abbas, and R. Murugesu, “( $\mu 1, \mu 2)$-pseudo almost automorphic solutions of fractional order neutral integrodifferential equations," Nonlinear Stud, vol. 24, pp. 669-685, 2017.

[66] V. Kavitha, S. Abbas, and R. Murugesu, "Asymptotically almost automorphic solutions of fractional order neutral integrodifferential equations," Bulletin of the Malaysian Mathematical Sciences Society, vol. 39, no. 3, pp. 1075-1088, 2016.

[67] S. Abbas, "Weighted pseudo almost automorphic solutions of fractional functional differential equations," Cubo (Temuco), vol. 16, pp. 21-35, 2014.

[68] E. Bazhlekova, Fractional Evolution Equations in Banach Spaces, Eindhoven University of Technology, 2001.

[69] S. D. Eidelman and A. N. Kochubei, "Cauchy problem for fractional diffusion equations," Journal of Differential Equations, vol. 199, no. 2, pp. 211-255, 2004.

[70] R. Gorenflo and F. Mainardi, "Fractional calculus: Integral and differential equations of fractional order," in Fractals and Fractional Calculus in Continuum Mechanics, A. Carpinteri and F. Mainardi, Eds., pp. 223-276, Springer-Verlag, NY, USA, Vienna, Austria, 1997.

[71] V. V. Anh and R. Mcvinish, "Fractional differential equations driven by Lévy noise," Journal of Applied Mathematics and Stochastic Analysis, vol. 16, no. 2, pp. 97-119, 2003.

[72] H.-S. Ding, J. Liang, and T.-J. Xiao, "Some properties of Stepanov-like almost automorphic functions and applications to abstract evolution equations," Applicable Analysis: An International Journal, vol. 88, no. 7, pp. 1079-1091, 2009.

[73] J. Liang, J. Zhang, and T.-J. Xiao, "Composition of pseudo almost automorphic and asymptotically almost automorphic functions," Journal of Mathematical Analysis and Applications, vol. 340, no. 2, pp. 1493-1499, 2008.

[74] M. Haase, "The functional calculus for sectorial operators," in Operator Theory: Advances and Applications, vol. 169, Birkhuser Verlag, Basel, Switzerland, 2006.

[75] E. Cuesta, "Asymptotic behaviour of the solutions of fractional integro-differential equations and some time discretizations," Discrete and Continuous Dynamical Systems - Series A, pp. 277285, 2007. 
[76] W. M. Ruess and W. H. Summers, "Compactness in spaces of vector valued continuous functions and asymptotic almost periodicity," Mathematische Nachrichten, vol. 135, pp. 7-33, 1988.

[77] D. R. Smart, Fixed Point Theorems, Cambridge University Press, London, UK, 1980. 


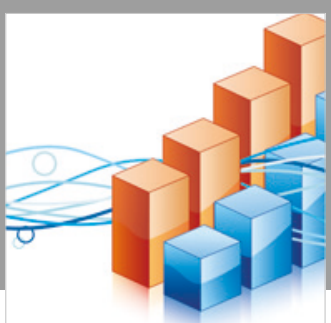

Advances in

Operations Research

\section{-n-m}
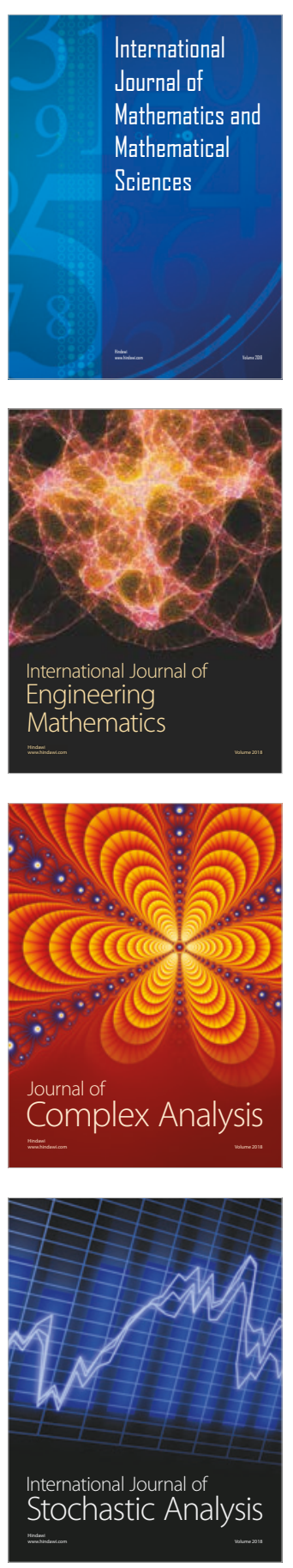
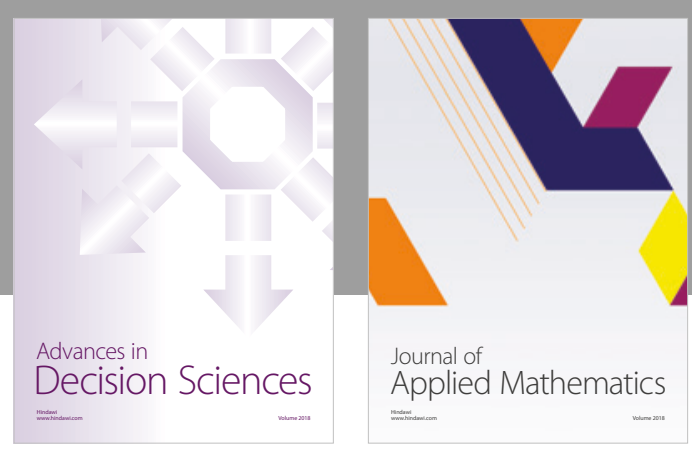

Journal of

Applied Mathematics
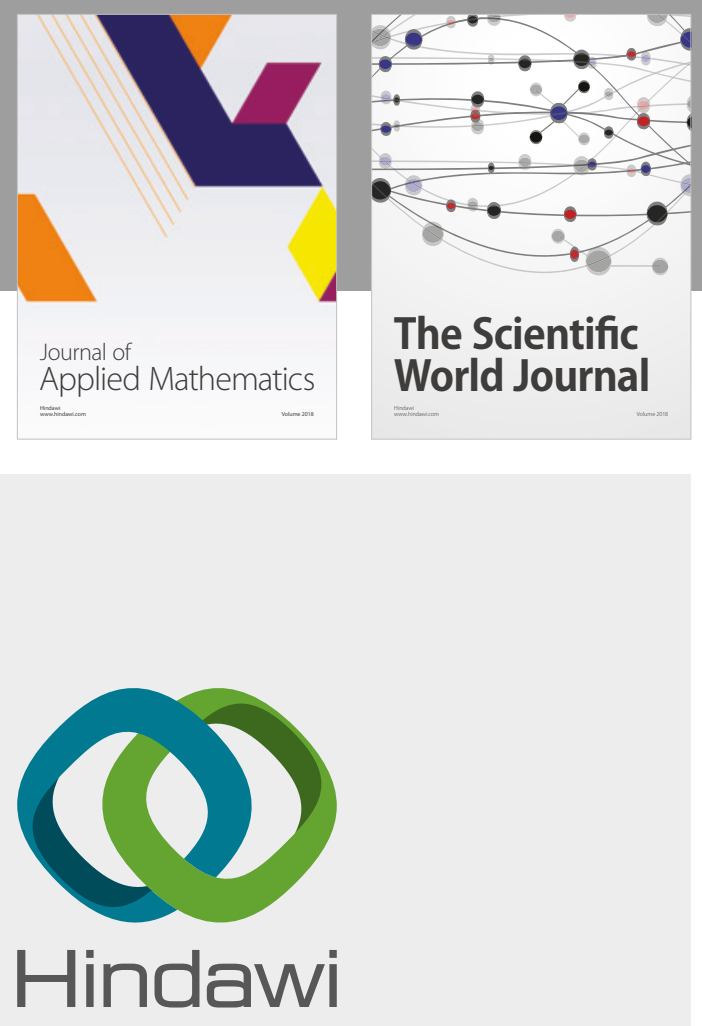

Submit your manuscripts at

www.hindawi.com

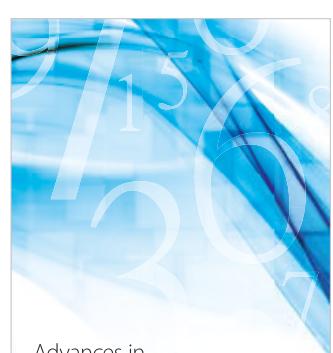

Advances in
Numerical Analysis
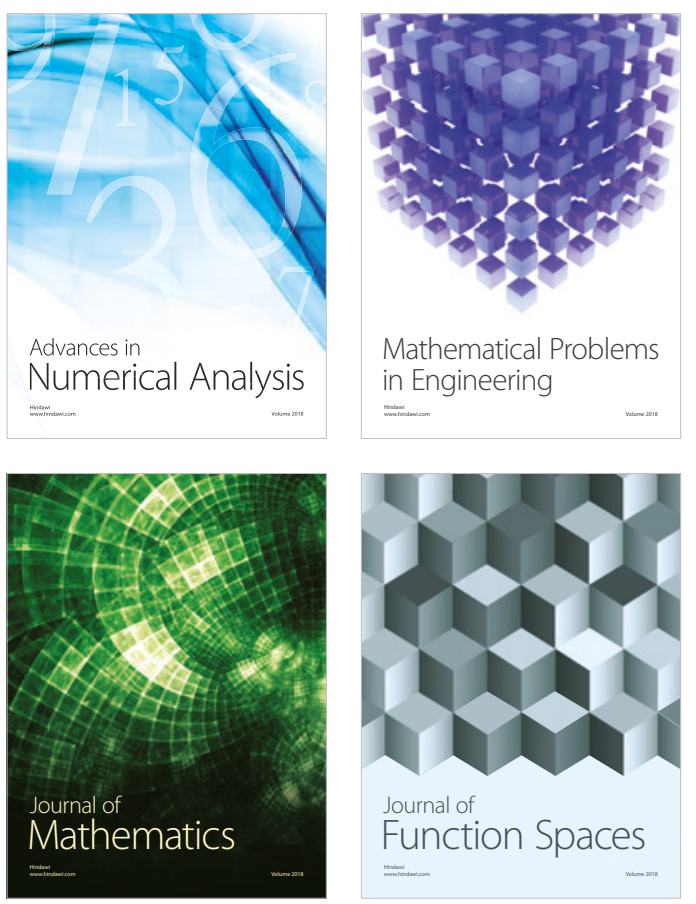

Mathematical Problems in Engineering

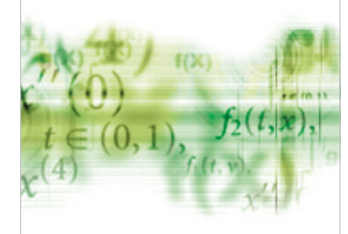

International Journal of

Differential Equations

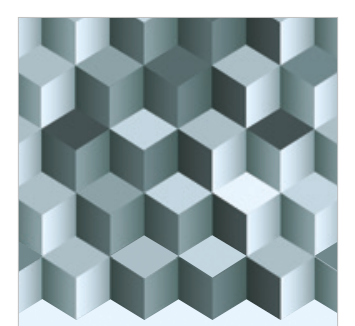

Journal of

Function Spaces

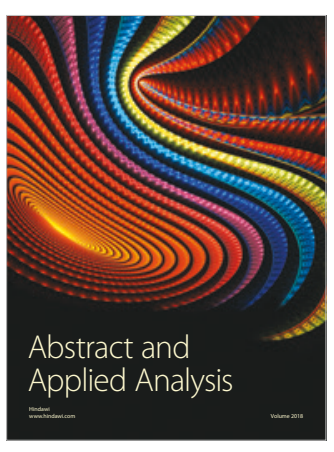

The Scientific

World Journal

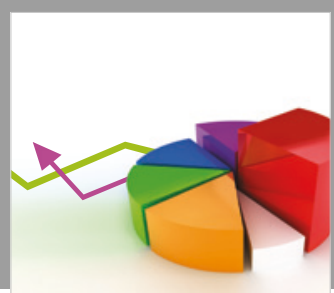

Journal of

Probability and Statistics
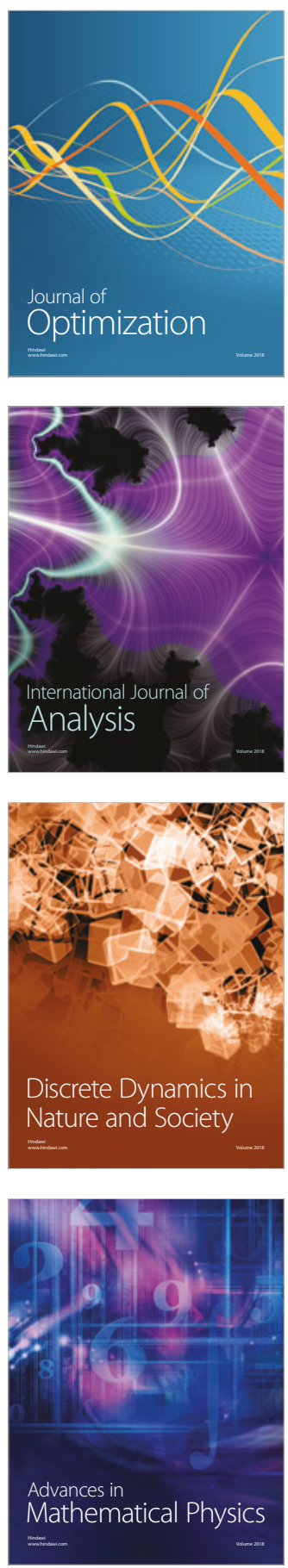